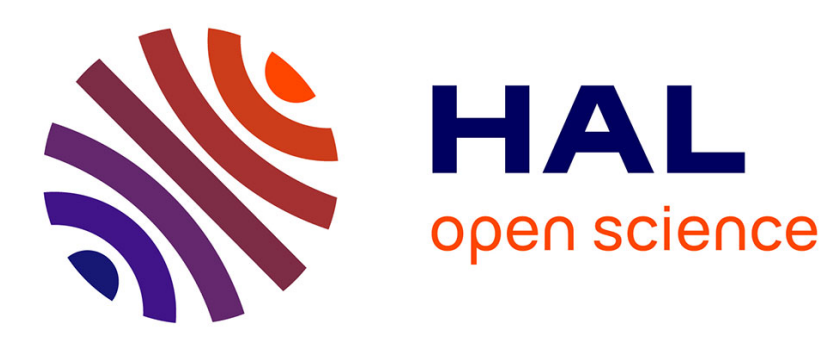

\title{
An Iterative Bayesian Algorithm for Sparse Component Analysis in Presence of Noise
}

Hadi Zayyani, Massoud Babaie-Zadeh, Christian Jutten

\section{To cite this version:}

Hadi Zayyani, Massoud Babaie-Zadeh, Christian Jutten. An Iterative Bayesian Algorithm for Sparse Component Analysis in Presence of Noise. IEEE Transactions on Signal Processing, 2009, 57 (11), pp.4378-4390. 10.1109/TSP.2009.2025154 . hal-00428706

\section{HAL Id: hal-00428706 https://hal.science/hal-00428706}

Submitted on 29 Oct 2009

HAL is a multi-disciplinary open access archive for the deposit and dissemination of scientific research documents, whether they are published or not. The documents may come from teaching and research institutions in France or abroad, or from public or private research centers.
L'archive ouverte pluridisciplinaire HAL, est destinée au dépôt et à la diffusion de documents scientifiques de niveau recherche, publiés ou non, émanant des établissements d'enseignement et de recherche français ou étrangers, des laboratoires publics ou privés. 


\title{
An Iterative Bayesian Algorithm for Sparse Component Analysis in Presence of Noise
}

\author{
Hadi Zayyani, Student Member, IEEE, Massoud Babaie-Zadeh, Senior Member, IEEE, and
} Christian Jutten, Fellow, IEEE

\begin{abstract}
We present a Bayesian approach for Sparse Component Analysis (SCA) in the noisy case. The algorithm is essentially a method for obtaining sufficiently sparse solutions of underdetermined systems of linear equations with additive Gaussian noise. In general, an underdetermined system of linear equations has infinitely many solutions. However, it has been shown that sufficiently sparse solutions can be uniquely identified. Our main objective is to find this unique solution. Our method is based on a novel estimation of source parameters and maximum $a$ posteriori (MAP) estimation of sources. To tackle the great complexity of the MAP algorithm (when the number of sources and mixtures become large), we propose an Iterative Bayesian Algorithm (IBA). This IBA algorithm is based on the MAP estimation of sources, too, but optimized with a steepest-ascent method. The convergence analysis of the IBA algorithm and its convergence to true global maximum are also proved. Simulation results show that the performance achieved by the IBA algorithm is among the best, while its complexity is rather high in comparison to other algorithms. Simulation results also show the low sensitivity of the IBA algorithm to its simulation parameters.
\end{abstract}

Index Terms-Atomic decomposition, blind source separation (BSS), sparse component analysis (SCA), sparse decomposition, sparse source separation.

\section{INTRODUCTION}

$\mathbf{F}$ INDING (sufficiently) sparse solutions of underdetermined systems of linear equations (possibly in the noisy case) has been studied extensively in recent years [1]-[7]. The problem has a growing range of applications in signal processing. To introduce the problem in more details, we will use the context of sparse component analysis (SCA) [8]. The discussions, however, may be easily followed in other contexts of application, for example, in finding a "sparse decomposition" of a signal on an overcomplete dictionary, which is the goal of the so-called overcomplete "atomic decomposition" [9]. Sparse representations are well suited for content analysis, i.e., extracting structure or meaning of a signal [10]. They may

Manuscript received January 23, 2008; accepted May 07, 2009. First published June 12, 2009; current version published October 14, 2009. The associate editor coordinating the review of this manuscript and approving it for publication was Prof. Marcelo G. S. Bruno. This work has been partially funded by Iran NSF (INSF) under contract number 86/994, by the Iran Telecom Research Center (ITRC), and also by the Center for International Research and Collaboration (ISMO) and the French embassy in Tehran in the framework of a GundiShapour collaboration program.

H. Zayyani and M. Babaie-Zadeh are with the Electrical Engineering Department and Advanced Communication Research Institute (ACRI), Sharif University of Technology, Tehran, Iran (e-mail: zayyani@ee.sharif.edu; mbzadeh@sharif.edu).

C. Jutten is with the GIPSA-lab, Grenoble, France, and the Institut Universitaire de France, France (e-mail: christian.jutten@ gipsa-lab.grenoble-inp.fr)

Digital Object Identifier 10.1109/TSP.2009.2025154 also be applied to signal compression applications to facilitate the storage, processing and communication of signals [11]. Another application of sparse decomposition is related to its denoising capability [12], [13]. Recently, interesting applications in decoding of real error correcting codes have been reported [14]-[16]. Also, some applications in the sampling theory have been initiated which can be regarded as unifying the sampling and the coding theories [17], [18].

SCA can be viewed as a method to achieve separation of sparse sources [3], [6]-[8], [19], [20]. The blind source separation (BSS) problem is to recover $m$ unknown sources from $n$ observed mixtures of them, where little or no information is available about the sources (except their statistical independence) and about the mixing system. In this paper, we consider the noisy linear instantaneous model at each instant:

$$
\mathbf{x}=\mathbf{A s}+\mathbf{n}
$$

where $\mathbf{x}, \mathbf{s}$ and $\mathbf{n}$ are $n \times 1, m \times 1$ and $n \times 1$ vectors of sources, mixtures and white Gaussian noises, respectively, and A is the $n \times m$ mixing matrix. In the underdetermined case $(m>n)$, estimating the mixing matrix is not sufficient to recover the sources, since the mixing matrix is not invertible. Then it appears that the estimation of sources requires other prior information on the sources. One prior information that can result in source recovery in underdetermined case is the sparsity of sources. The sparsity of a source vector means that almost all its entries are zero (or near zero) and only a few entries are nonzero. If we restrict ourselves to sufficiently sparse solutions of the underdetermined system of linear equations, it has been shown that the solution is unique [1], [2], [21].

SCA can be solved in two steps: first estimating the mixing matrix, and then estimating the sources. The first step may be accomplished by means of clustering [3], [22] or other methods [6], [7]. The second step requires finding the sparse solution of (1) assuming $\mathbf{A}$ to be known [23]-[27]. Finally, some methods estimate the mixing matrix and sources simultaneously [5], [20]. In this paper, we focus on the source estimation, assuming $\mathbf{A}$ is already known.

Atomic decomposition [9] is another viewpoint to the same mathematical problem as above. In this viewpoint, we have just "one" signal whose samples are collected in the $n \times 1$ signal vector $\mathbf{X}$ and the objective is to express it as a linear combination of a set of predetermined signals where their samples are collected in vectors $\left\{\mathbf{a}_{i}\right\}_{i=1}^{m}$. After [28], the $\mathbf{a}_{i}$ 's are called atoms and they collectively form a dictionary over which the signal is to be decomposed. In this paper, we also consider an additive noise term in the decomposition. So we can write $\mathbf{x}=\mathbf{A s}+\mathbf{n}$, 
where $\mathbf{A}$ is the $n \times m$ dictionary (matrix) with the columns being the atoms and $\mathbf{s}$ is the $m \times 1$ vector of coefficients. The vector $\mathbf{n}$ can be interpreted either as the noisy term of the original signal that we intend to decompose (the term that is not in the span of our dictionary) or as the allowed error for the decomposition process. A dictionary with $m>n$ atoms is called overcomplete. It is clear that the representation of a signal on an overcomplete dictionary is not unique. However, as above, if a signal has a sufficiently sparse representation over such a dictionary, it would be unique under some mild conditions on the dictionary [4].

To obtain the sparse solution of (1), an approach is to search for solutions having minimum $\ell^{0}$-norm, i.e., minimum number of nonzero components. This method is computationally intractable when the dimension increases (due to the need for a combinatorial search), and it is too sensitive to noise (because any small amount of noise completely change the $\ell^{0}$-norm of a vector). Then, one of the most successful approaches of finding the sparse solution is Basis Pursuit (BP) [9], which achieves a convexification of the problem by replacing the $\ell^{0}$-norm with an $\ell^{1}$-norm. In other words, BP proposes to find the solution of (1) for which $\sum_{i=1}^{m}\left|s_{i}\right|$ is minimized. The minimum $\ell^{1}$-norm solution is also the maximum a posteriori (MAP) source estimation under Laplacian source prior for the noiseless case [29]. As a main benefit, it can be easily implemented by linear programming (LP) methods (especially fast interior-point LP solvers). Recently, a fast method called smoothed $-\ell^{0}$ method has been proposed to minimize a smoothed approximation of the $\ell^{0}$-norm [25], [26]. The FOCal Underdetermined System Solver (FOCUSS) uses $\ell^{p}$-norm as a replacement for the $\ell^{0}$-norm and a Lagrange multiplier method for the optimization [21]. Other simple approaches are Matching Pursuit (MP) [28], [30] and Orthogonal Matching Pursuit (OMP) [31] algorithms. Another refined algorithm is the stage-wise Orthogonal Matching Pursuit (stOMP) [32]. Recently, a fast iterative detection-estimation algorithm has been used to solve this problem [23]. Moreover, Bayesian approaches have been proposed: Sparse Bayesian Learning (SBL) [33] and recently, Bayesian Compressive Sensing (BCS) [34]. Finally, sparse reconstruction are possible using some new gradient methods like gradient pursuits [35] and gradient projection [36].

Among available methods for sparse decomposition, the fast methods (e.g., MP) usually do not produce accurate results, while $\mathrm{BP}$ which is guaranteed to asymptotically obtain the exact minimum $\ell^{0}$-norm solution will become computationally demanding for large dimensions. With regard to the new applications of this area in decoding [14] or denoising [12] in which the performance of the algorithm in presence of noise is very important, finding a new algorithm with a better accuracy is a necessity. In this paper, our main concern is in this way.

In our algorithm, we first proposed a three-step (sub-)optimum (in the MAP sense) method for SCA in the noisy underdetermined case for Bernoulli-Gaussian (BG) sources. It has the drawback of large complexity which is not tractable when there are many sources and mixtures. To tackle the great complexity, an iterative method for MAP estimation of sources is presented. This leads to a more efficient algorithm, which is again a three-step iterative Bayesian algorithm, among which two steps are expectation and maximization steps. These steps resemble the classical EM algorithm [37] which is also successfully used in [38].

BG sources is a usual model in the field of deconvolution, which is reviewed by Mendel in [39], and it was used especially in seismic deconvolution [39]-[43]. Using $\ell^{1}$-norm to find sparse solutions of linear systems of equations can also be traced back to Taylor et al. [40] in this field. The relaxation of the discrete nature of BG processes by using a Gaussian mixture model has been considered in [43], based on a cost function with a weighting parameter. The authors used an EM algorithm for estimating their parameters, with a heuristic method for choosing the weighting parameter. In contrast, our work is originated from a MAP algorithm and the treatment is totally Bayesian and the cost function is dependent on the statistical parameters. We also used iterative Bayesian techniques to estimate all the unknowns in a rather unified way. So, the global algorithm resembles an EM algorithm, while we used some other Bayesian techniques for parameter estimation.

Our approach can be categorized as a Bayesian method for SCA [5], [20], [44] which is efficient for large number of sources and mixtures.

The paper starts with the statement of the system model in Section II. Then, a MAP solution is proposed in Section III. Based on this algorithm, we develop the new Iterative Bayesian Algorithm (IBA) in Section IV. Section V provides proof of convergence of the proposed IBA algorithm. Finally, we present some simulation results in Section VI.

\section{SySTEM MODEL}

The noise vector in the model (1) is assumed zero-mean Gaussian with a covariance matrix $\sigma_{n}^{2} \mathbf{I}$. For modeling the sparse sources, we assumed the sources are inactive with probability $p$, and are active with probability $1-p$ (sparsity of sources implies that $p$ is near 1). In the inactive case, the value of sources is zero and in the active case the value is obtained from a Gaussian distribution. This is called a Bernoulli-Gaussian model. The BG model is a realistic model in the sparse deconvolution applications and it has been extensively used in this literature [39]-[43]. This model has also been used in [16] for simultaneously modeling the impulse and background noise in a real-field channel coding system. It is also used to model the impulse noise in a communication channel [45], or the sparse vector in an application of regression [46]. This model is also suitable for sparse decomposition applications where we want to decompose a signal as a combination of only a few atoms of the dictionary, while the coefficients of the other atoms are zero. So, the probability density of the sources in SCA (or coefficients in sparse decomposition) is

$$
p\left(s_{i}\right)=p \delta\left(s_{i}\right)+(1-p) N\left(0, \sigma_{r}^{2}\right) .
$$

In this model, any sample of the sources can be written as $s_{i}=q_{i} r_{i}$ where $q_{i}$ is a binary variable (with binomial distribution) and $r_{i}$ is the amplitude of the $i$ th source with Gaussian distribution. So the source vector can be written as

$$
\mathbf{s}=\mathbf{Q r}, \quad \mathbf{Q}=\operatorname{diag}(\mathbf{q})
$$




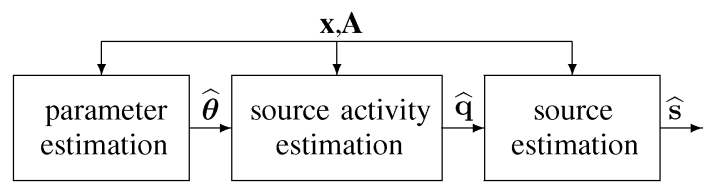

Fig. 1. Block diagram of our MAP algorithm.

where $\mathbf{q} \triangleq\left(q_{1}, q_{2}, \ldots, q_{m}\right)^{T}$ and $\mathbf{r} \triangleq\left(r_{1}, r_{2}, \ldots, r_{m}\right)^{T}$ are the "source activity vector" and "amplitude vector," respectively. Each element of the source activity vector shows the activity of the corresponding source. That is

$$
q_{i}= \begin{cases}1 & \text { if } \left.s_{i} \text { is active (with probability } 1-p\right) \\ 0 & \text { if } s_{i} \text { is inactive (with probability } p \text { ) }\end{cases}
$$

Consequently, the probability $p(\mathbf{q})$ of source activity vector $\mathbf{q}$ is equal to

$$
p(\mathbf{q})=(1-p)^{n_{a}}(p)^{m-n_{a}}
$$

where $n_{a}$ is the number of active sources i.e., the number of 1's in q. Note that, in this paper, we use the same notation $p($.$) for$ both the probability and for Probability Density Function (PDF).

\section{MAP ALGORITHM}

In [24], we proposed a three-step MAP algorithm for the noisy sparse component analysis. Here, we explain it in details, because it is the basis of our IBA algorithm. Its block diagram is shown in Fig. 1, and consists of three steps. The first step is the estimation of the source parameters $\left(p, \sigma_{r}\right)$ and noise parameter $\left(\sigma_{n}\right)$. If we define the parameter vector as $\boldsymbol{\theta}=\left(p, \sigma_{r}, \sigma_{n}\right)^{T}$, the objective of this step is to obtain an estimation of $\boldsymbol{\theta}$. The second step is the estimation of the source activity vector $(\mathbf{q})$ which is defined in (4). The last step is the source estimation.

\section{A. Parameter Estimation}

The parameter estimation step is done by a novel method based on second and fourth order moments (for $p$ and $\sigma_{r}$ ) and by a special application of EM algorithm [47] (for $\sigma_{n}$ ). For estimating $p$ and $\sigma_{r}$, let one of the mixtures, be $x=\sum_{i=1}^{m} a_{i} s_{i}+$ $n=y+n$. Then, neglecting the noise contribution (i.e., assuming $\sigma_{n} \ll \sigma_{r}$ ), and taking into account that the sources are independent and zero-mean, then the relationship between the moments of the mixture and the moments of the sources are

$$
\begin{aligned}
E\left(x^{2}\right) \approx & E\left(y^{2}\right)=\left(\sum_{i} a_{i}^{2}\right) E\left(s_{i}^{2}\right) \\
E\left(x^{4}\right) \approx & E\left(y^{4}\right)=\left(\sum_{i} a_{i}^{4}\right) E\left(s_{i}^{4}\right) \\
& +\left(6 \sum_{i, j, i \neq j} a_{i}^{2} a_{j}^{2}\right) E^{2}\left(s_{i}^{2}\right) .
\end{aligned}
$$

In (6) and (7), we have assumed that the noise term is small and so the moments of the mixture without noise $(y)$ are approximately the same as the moment of the true mixture $(x)$. From the above equations and by assuming that all the sources have equal moments (due to identical parameters) and the $a_{i}$ 's are known, the source moments can be computed. Especially, concerning the second and fourth order moments of the sources, one can obtain the following relations:

$$
\begin{aligned}
& m_{2}=E\left(s_{i}^{2}\right)=(1-p) \sigma_{r}^{2} \\
& m_{4}=E\left(s_{i}^{4}\right)=3(1-p) \sigma_{r}^{4} .
\end{aligned}
$$

From (8) and (9), we can compute the two source parameters:

$$
\begin{aligned}
\hat{\sigma}_{r} & =\sqrt{\frac{\hat{m}_{4}}{3 \hat{m}_{2}}} \\
\hat{p} & =1-\frac{3 \hat{m}_{2}^{2}}{\hat{m}_{4}}
\end{aligned}
$$

where $\hat{m}_{2}$ and $\hat{m}_{4}$ are estimated by (6) and (7). Moreover, $E\left(x^{2}\right)$ and $E\left(x^{4}\right)$ are estimated from empirical expectation (weighted sum) of the corresponding mixture. To estimate the observation noise variance $\left(\sigma_{n}\right)$, we use a special application of EM algorithm introduced in [47] which gives a maximum likelihood estimation of parameters of a Mixture of Gaussian (MoG) (centers, probabilities, and variances) distribution from its samples. Since each $s_{i}$ can be either active or inactive, a mixture $x=\sum_{i=1}^{m} a_{i} s_{i}+n$ can be modeled by a mixture of $2^{m}$ Gaussian components. Since $p$ is close to one $(p \approx 1)$, one can neglect powers of $(1-p)$ greater than one, which leads to the following approximation of noisy sparse MoG:

$$
\begin{aligned}
p(x) \approx(p)^{m} N\left(0, \sigma_{n}^{2}\right)+(p)^{(m-1)}(1-p) & \\
& \times \sum_{i=1}^{m} N\left(0, a_{i}^{2} \sigma_{r}^{2}+\sigma_{n}^{2}\right) .
\end{aligned}
$$

This equation shows that each mixture has a MoG distribution and the smallest variance of these Gaussians is the variance of the noise $\left(\sigma_{n}\right)$. Consequently, for estimating $\sigma_{n}$, the EM algorithm in [47] is applied to one of the mixture signals.

\section{B. Estimation of the Source Activity Vector}

To estimate the source activity vector $(\mathbf{q})$, we use the MAP detector which maximizes the posterior probability $p(\mathbf{q} \mid \mathbf{x})$. Using the Bayes' rule:

$$
p(\mathbf{q} \mid \mathbf{x})=\frac{p(\mathbf{q}) p(\mathbf{x} \mid \mathbf{q})}{p(\mathbf{x})}
$$

the MAP detector should maximize $p(\mathbf{q}) p(\mathbf{x} \mid \mathbf{q})$. The prior source activity probability $(p(\mathbf{q}))$ is given by (5). The likelihood $(p(\mathbf{x} \mid \mathbf{q}))$ has a Gaussian distribution with the following conditional covariance:

$$
\mathbf{Q}_{q}=E\left(\mathbf{x x}^{T} \mid \mathbf{q}\right)=\mathbf{A} \mathbf{V}_{q} \mathbf{A}^{T}+\sigma_{n}^{2} \mathbf{I}
$$

where the matrix $\mathbf{V}_{q}$ is the conditional covariance of the sources and can be stated as

$$
\mathbf{V}_{q}=\sigma_{r}^{2} \mathbf{Q}
$$

where $\mathbf{Q}$ was defined in (3). Consequently, the prior probability $p(\mathbf{x} \mid \mathbf{q})$ has the following Gaussian form:

$$
p(\mathbf{x} \mid \mathbf{q})=\frac{1}{\sqrt{\operatorname{det}\left(2 \pi \mathbf{Q}_{q}\right)}} \exp \left(\frac{-1}{2} \mathbf{x}^{T} \mathbf{Q}_{q}^{-1} \mathbf{x}\right)
$$


and the MAP detector maximizes the $p(\mathbf{q}) p(\mathbf{x} \mid \mathbf{q})$ over all $2^{m}$ cases for all source activity vectors:

$$
\mathbf{q}_{\mathrm{MAP}}=\underset{\mathbf{q}}{\arg \max } \frac{p(\mathbf{q})}{\sqrt{\operatorname{det}\left(2 \pi \mathbf{Q}_{q}\right)}} \exp \left(\frac{-1}{2} \mathbf{x}^{T} \mathbf{Q}_{q}^{-1} \mathbf{x}\right) .
$$

\section{Estimation of Sources}

After estimating the source activity vector, the estimation of sources is achieved with the estimation of amplitudes $\left(r_{i}\right.$ 's), i.e., of amplitude vector $(\mathbf{r})$. The vector $\mathbf{r}$ has a Gaussian distribution and hence its MAP estimation is equal to its linear least square (LLS) estimation [48]. The LLS estimation of $\mathbf{r}$ by knowing the source activity vector and the mixture vector has the following form [48]:

$$
\widehat{\mathbf{r}}_{\mathrm{LLS}}=E\left(\mathbf{r} \mathbf{x}^{T} \mid \mathbf{q}\right) E\left(\mathbf{x} \mathbf{x}^{T} \mid \mathbf{q}\right)^{-1} \mathbf{x} .
$$

The calculation of the two terms $E\left(\mathbf{r} \mathbf{x}^{T} \mid \mathbf{q}\right)$ and $E\left(\mathbf{x x}^{T} \mid \mathbf{q}\right)$ results in the following equations:

$$
\begin{aligned}
& E\left(\mathbf{r x} \mathbf{x}^{T} \mid \mathbf{q}\right)=\sigma_{r}^{2} \mathbf{Q} \mathbf{A}^{T} \\
& E\left(\mathbf{x x}^{T} \mid \mathbf{q}\right)=\sigma_{r}^{2} \mathbf{A} \mathbf{Q} \mathbf{A}^{T}+\sigma_{n}^{2} \mathbf{I} .
\end{aligned}
$$

Then, the source amplitudes are estimated as

$$
\widehat{\mathbf{r}}=\sigma_{r}^{2} \mathbf{Q} \mathbf{A}^{T}\left(\sigma_{r}^{2} \mathbf{A} \mathbf{Q} \mathbf{A}^{T}+\sigma_{n}^{2} \mathbf{I}\right)^{-1} \mathbf{x} .
$$

The maximization of $p(\mathbf{q}) p(\mathbf{x} \mid \mathbf{q})$ may be done by exhaustive search over the discrete space of vectors $\mathbf{q}$ with $2^{m}$ discrete elements. This method can solve the SCA problem only for a small number of sources (for example $m<12$ ). However, the complexity of this algorithm, based on an exhaustive search, can be alleviated with the IBA algorithm described in the next section.

\section{ITERATIVE BAYESIAN ALGORITHM}

\section{A. Basic Idea}

In the MAP algorithm, the maximization of the posterior probability given by (17) is done by a combinatorial search over the discrete space. In this section, we propose a maximization method based on first converting the problem to a continuous maximization and then using a steepest-ascent algorithm. In this purpose, we use a mixture of two Gaussian model centered around 0 and 1 with sufficiently small variances. Thus, the discrete binomial variable $q_{i}$ is converted to a variable with the following form:

$$
q_{i} \sim p N\left(0, \sigma_{0}^{2}\right)+(1-p) N\left(1, \sigma_{0}^{2}\right) .
$$

To avoid local maxima of (17) a gradually decreasing variance is used in the different iterations (this is similar to what is done in simulated annealing algorithms, and to graduated nonconvexity [49]). However, (17), as a cost function with respect to $\mathbf{q}$, is very complex to work with.

The main idea of our algorithm is that the source estimation is equivalent to estimation of vectors $\mathbf{q}$ and $\mathbf{r}$ (as observed from (3)) and can be done iteratively. First, an estimated vector $\widehat{\mathbf{q}}$ is assumed and then the MAP estimate of the vector $\mathbf{r}$ based on the known $\widehat{\mathbf{q}}$ and the observation vector $\mathbf{x}$ is obtained (we refer to it as $\widehat{\mathbf{r}})$. Secondly, the MAP estimate of vector $\mathbf{q}$ is obtained based on estimated vector $\widehat{\mathbf{r}}$ and observation vector $\mathbf{x}$ (we refer to it as vector $\widehat{\mathbf{q}}$ ). Therefore, the MAP estimation of sources is achieved through two other elementary MAP estimation steps.

In the first step, a source activity vector $\widehat{\mathbf{q}}$ is assumed and the estimation of $\mathbf{r}$ will be computed. Because the vector $\mathbf{r}$ is Gaussian, its MAP estimate is equal to the LLS estimation [48] and can be computed as follows:

$$
\widehat{\mathbf{r}}_{\mathrm{MAP}}=\widehat{\mathbf{r}}_{\mathrm{LLS}}=E(\mathbf{r} \mid \mathbf{x}, \widehat{\mathbf{q}})=E\left(\mathbf{r} \mathbf{x}^{T} \mid \widehat{\mathbf{q}}\right) E\left(\mathbf{x} \mathbf{x}^{T} \mid \widehat{\mathbf{q}}\right)^{-1} \mathbf{x} .
$$

This step can be called Expectation step or Estimation step (E-step). Computation and simplification of (23) (similar to what done in [24]) leads to the following equation which is similar to (21):

$$
\widehat{\mathbf{r}}=\sigma_{r}^{2} \widehat{\mathbf{Q}} \mathbf{A}^{T}\left(\sigma_{r}^{2} \mathbf{A} \widehat{\mathbf{Q}} \mathbf{A}^{T}+\sigma_{n}^{2} \mathbf{I}\right)^{-1} \mathbf{x} .
$$

In the second step which can be called Maximization step (M-step), we estimate $\mathbf{q}$ based on known $\widehat{\mathbf{r}}$ and observed $\mathbf{x}$. The MAP estimation is

$$
\begin{aligned}
\widehat{\mathbf{q}}_{\mathrm{MAP}} & =\underset{\mathbf{q}}{\arg \max } p(\mathbf{q} \mid \mathbf{x}, \widehat{\mathbf{r}}) \equiv \underset{\mathbf{q}}{\arg \max } p(\mathbf{q} \mid \widehat{\mathbf{r}}) p(\mathbf{x} \mid \mathbf{q}, \widehat{\mathbf{r}}) \\
& \equiv \underset{\mathbf{q}}{\arg \max } p(\mathbf{q}) p(\mathbf{x} \mid \mathbf{q}, \widehat{\mathbf{r}}) \\
& \equiv \underset{\mathbf{q}}{\arg \max }(\log p(\mathbf{q})+\log p(\mathbf{x} \mid \mathbf{q}, \widehat{\mathbf{r}})) .
\end{aligned}
$$

Equation (22) implies that, in (25) $p(\mathbf{q})$ can be computed as a continuous variable:

$$
\begin{aligned}
p(\mathbf{q}) & =\prod_{i=1}^{m} p\left(q_{i}\right) \\
& =\prod_{i=1}^{m}\left[p \exp \left(\frac{-q_{i}^{2}}{2 \sigma_{0}^{2}}\right)+(1-p) \exp \left(\frac{-\left(q_{i}-1\right)^{2}}{2 \sigma_{0}^{2}}\right)\right]
\end{aligned}
$$

In addition, the term $p(\mathbf{x} \mid \mathbf{q}, \widehat{\mathbf{r}})$ in (25) can be computed as

$$
\begin{aligned}
p(\mathbf{x} \mid \mathbf{q}, \widehat{\mathbf{r}})= & p_{n}(\mathbf{x}-\mathbf{A Q} \widehat{\mathbf{r}}) \\
= & \left(2 \pi \sigma_{n}^{2}\right)^{\frac{-m}{2}} \\
& \times \exp \left(\frac{-1}{2 \sigma_{n}^{2}}(\mathbf{x}-\mathbf{A Q} \widehat{\mathbf{r}})^{T}(\mathbf{x}-\mathbf{A Q} \widehat{\mathbf{r}})\right) .
\end{aligned}
$$

Consequently, (25) writes as

$$
\mathrm{M}-\text { step }: \quad \widehat{\mathbf{q}}=\underset{q}{\arg \max } L(\mathbf{q})
$$

where

$$
L(\mathbf{q})=\sum_{i=1}^{m} \log \left(p\left(q_{i}\right)\right)-\frac{1}{2 \sigma_{n}^{2}}(\mathbf{x}-\mathbf{A Q} \widehat{\mathbf{r}})^{T}(\mathbf{x}-\mathbf{A} \mathbf{Q} \widehat{\mathbf{r}}) .
$$

Maximization of $L(\mathbf{q})$ in the M-step can be done by the steepest-ascent method. The main steepest-ascent iteration is

$$
\mathbf{q}_{k+1}=\mathbf{q}_{k}+\mu \frac{\partial L(\mathbf{q})}{\partial \mathbf{q}}
$$


where $\mu$ is the step-size in the steepest-ascent method. After calculating the gradient (see Appendix I), the steepest-ascent algorithm for the M-step is

$$
\mathbf{q}_{k+1}=\mathbf{q}_{k}+\frac{\mu}{\sigma_{0}^{2}} \mathbf{g}(\mathbf{q})+\frac{\mu}{\sigma_{n}^{2}} \operatorname{diag}\left(\mathbf{A}^{T}(\mathbf{A Q} \widehat{\mathbf{r}}-\mathbf{x})\right) \cdot \widehat{\mathbf{r}}
$$

where $\mathbf{g}(\mathbf{q})$, given in Appendix I by (58), depends on $\sigma_{0}$. In successive iterations of our simulations, we gradually decrease the variance $\sigma_{0}$ according to the series $\sigma_{0}^{(i)}=\alpha \sigma_{0}^{(i-1)}$ where $\alpha$ is selected between 0.6 and 1. In Section V, a suitable range on values of $\mu$ for the convergence of the proposed IBA algorithm is calculated. This suitable interval means that if the step size $\mu$ is selected in this interval, with a probability close to one, the convergence is insured. Assuming the columns of $\mathbf{A}$ are normalized to have unit norms, the suitable interval is

$$
0<\mu<\frac{2}{\frac{1}{\sigma_{0}^{2}}+\frac{m M^{* 2}}{\sigma_{n}^{2}}}
$$

where $M^{*}=\sigma_{r} Q^{-1}\left(\frac{1-\sqrt[m]{0.99}}{2}\right)$. As we see from (31) the second summand is responsible for increasing the prior probability $p(\mathbf{q})$ while the third summand is responsible for decreasing the noise power $\|\mathbf{x}-\mathbf{A s}\|^{2}$. When $\sigma_{0}$ is much larger than $\sigma_{n}$, the second term is weaker than the third term and as a result the exactness of $\mathbf{x}=\mathbf{A s}$ is more relevant than the sparsity of $\mathbf{s}$. When $\sigma_{0}$ is comparable to $\sigma_{n}$, both terms are effective to yield the equilibrium point between sparsity and noise.

In summary, the overall algorithm is a three-step iterative algorithm the first two steps of which are E-step and M-step in (24) and (31), while the last step, explained in the next section, is the parameter estimation step.

\section{B. Parameter Estimation}

So far the parameters $\left(p, \sigma_{r}\right.$ and $\left.\sigma_{n}\right)$ are assumed to be known. The parameter estimation is necessary and can also be done iteratively. We also assume that the columns of the matrix have unit norms. In other words, the basis atoms of our dictionary are normalized to have unit norm. With these assumptions and by assuming the ergodicity of sources (i.e., the mixtures can be considered as samples of a random variable $x_{j}=\sum_{i=1}^{m} a_{j i} s_{i}+e_{j}$ where $a_{j i}=b_{j i} / \sqrt{b_{1 i}^{2}+b_{2 i}^{2}+\ldots+b_{n i}^{2}}$ and $b_{j i}$ is a random variable with uniform distribution on $[-1,1]$ and $s_{i}$ and $e_{j}$ are random variables), and by neglecting the noise power, we have $E\left(x_{j}^{2}\right)=m E\left(a_{j i}^{2}\right) E\left(s_{i}^{2}\right)$. In addition, we know that $\sum_{j=1}^{n} a_{j i}^{2}=1$ and hence we deduce $E\left(a_{j i}^{2}\right)=1 / n$. Finally, from (8), we have $E\left(s_{i}^{2}\right)=(1-p) \sigma_{r}^{2}$, and therefore we can write

$$
\widehat{\sigma_{r}}=\sqrt{\frac{n E\left(x_{j}^{2}\right)}{m(1-\widehat{p})}}
$$

With the initialization of $\widehat{p}$ with $\hat{p}^{(0)}$, we will have $\widehat{\sigma_{r}}=\sqrt{n E\left(x_{j}^{2}\right) / m\left(1-\widehat{p}^{(0)}\right)}$. For the starting noise variance, we choose ${\widehat{\sigma_{n}}}^{(0)}=\widehat{\sigma_{r}} / 10$. In [27], we used the algorithm with a simple non iterative parameter estimation step that was stated above. But, here these simple non iterative estimates are just used as the initial estimation of the parameters. Along the
- Initialization:

1) Let $\boldsymbol{\theta}_{0}$ equal to the initial parameter estimation:

$\hat{p}^{(0)}$ : arbitrary value in $[0.51]$,

$\hat{\sigma}_{r}^{(0)}=\sqrt{\frac{n E\left(x_{j}^{2}\right)}{m\left(1-\widehat{p}^{(0)}\right)}}, \hat{\sigma}_{n}^{(0)}=\frac{\hat{\sigma}_{r}^{(0)}}{10}$.

2) Let $\mathbf{s}_{0}, \mathbf{q}_{0}$ and $\mathbf{r}_{0}$ equal to the initial solution from minimum $\ell^{2}$-norm: $\mathbf{s}_{0}=\mathbf{A}^{T}\left(\mathbf{A A}^{T}\right)^{-1} \mathbf{x}$

$\mathbf{q}_{0}=\left(\mathbf{s}_{0}>T h\right), \mathbf{r}_{0}=\mathbf{s}_{0} \cdot\left(\mathbf{s}_{0}>T h\right)$.

- Until Convergence do:

1) E-step: solution obtained in (24).

2) M-step:

- for $j=1, \ldots, n_{\text {iteration }}$

* Update q with (31)

* Update $\sigma_{0}^{(j)}=\alpha \sigma_{0}^{(j-1)}$

3) Parameter Estimation Step: using (33), (34), (35) and (36).

- Final answer is $\hat{\mathbf{s}}=\mathbf{q}_{\mathrm{final}} \mathbf{r}_{\mathrm{final}}$.

Fig. 2. Final overall IBA algorithm estimation.

iterations, we update the estimation of these parameters using the following simple equations:

$$
\begin{aligned}
\widehat{p} & =\frac{\|\mathbf{q}\|_{0}}{m} \\
\widehat{\sigma_{n}} & =\frac{\|\mathbf{x}-\mathbf{A} \widehat{\mathbf{s}}\|_{2}}{\sqrt{n}} \\
\widehat{\sigma_{r}} & =\frac{\|\mathbf{r}\|_{2}}{\sqrt{m}}
\end{aligned}
$$

where it is assumed that the number of sources $m$ is known in advance. In addition to (36), we can use (33) to update the estimation of $\sigma_{r}$ in the iterations.

It is shown in Appendix II that the formulas (34), (35), and (36) are the MAP estimates of the corresponding parameters based on knowing all the other unknowns.

Finally, the complete IBA algorithm, including the parameter estimation step, is summarized in Fig. 2. Since the active sources are Gaussian with a variance $\sigma_{r}^{2}$, we choose the threshold parameter $T h$ as a fraction of $\sigma_{r}$. The exact value of $T h$ determines the number of active sources in the first step. We will discuss about this parameter in the simulation results section.

\section{Convergence Analysis}

In this section, we first prove that the log posterior probability $L(\mathbf{s}) \triangleq \log p(\mathbf{s} \mid \mathbf{x})$ is concave. Therefore, the unique maximum of this function is the global maximum. Then, we prove the convergence of the steepest-ascent algorithm in the M-step and find the suitable interval for the step-size $(\mu)$ for the convergence of the steepest-ascent algorithm. Finally, the convergence of the overall IBA algorithm will be proved.

\section{A. Concavity of the Posterior Probability}

The $\log$ posterior probability function $L(\mathbf{s})$ can be written as $L(\mathbf{s}) \propto \log p(\mathbf{s})+\log p(\mathbf{x} \mid \mathbf{s})$, that we can decompose as

$$
L(\mathbf{s})=\sum_{i=1}^{m} \log \left(p\left(s_{i}\right)\right)-\frac{1}{2 \sigma_{n}^{2}}(\mathbf{x}-\mathbf{A} \mathbf{s})^{T}(\mathbf{x}-\mathbf{A} \mathbf{s}) .
$$


To prove the concavity of $L(\mathbf{s})$, we prove the concavity of each $\log \left(p\left(s_{i}\right)\right)$ and the convexity of $c(\mathbf{s}) \triangleq(\mathbf{x}-\mathbf{A} \mathbf{s})^{T}(\mathbf{x}-\mathbf{A} \mathbf{s})$. Then, since the sum of concave functions is also concave, the proof is completed. As before, the probability distribution of sources in (2) is assumed to be a BG distribution

$$
p\left(s_{i}\right)=\frac{p}{\sigma_{1} \sqrt{2 \pi}} \exp \left(\frac{-s_{i}^{2}}{2 \sigma_{1}^{2}}\right)+\frac{1-p}{\sigma_{2} \sqrt{2 \pi}} \exp \left(\frac{-s_{i}^{2}}{2 \sigma_{2}^{2}}\right)
$$

with sufficiently small $\sigma_{1}$. To prove the concavity of $\log \left(p\left(s_{i}\right)\right)$, we need to prove that its second derivative is negative. It is equivalent to prove that $h\left(s_{i}\right) \triangleq p\left(s_{i}\right) p^{\prime \prime}\left(s_{i}\right)-p^{\prime}\left(s_{i}\right)^{2}<0$. Calculation of this function leads to the following formula:

$$
\begin{aligned}
\frac{1}{2 \pi} h\left(s_{i}\right)= & -\frac{p^{2}}{\sigma_{1}^{4}} \exp \left(\frac{-s_{i}^{2}}{\sigma_{1}^{2}}\right)-\frac{(1-p)^{2}}{\sigma_{2}^{4}} \exp \left(\frac{-s_{i}^{2}}{\sigma_{2}^{2}}\right) \\
& +\frac{p(1-p)}{\sigma_{1} \sigma_{2}}\left[\left(\frac{s_{i}}{\sigma_{1}^{2}}-\frac{s_{i}}{\sigma_{2}^{2}}\right)^{2}-\frac{1}{\sigma_{1}^{2}}-\frac{1}{\sigma_{2}^{2}}\right] \\
& \times \exp \left(\frac{-s_{i}^{2}}{2 \sigma_{1}^{2}}-\frac{-s_{i}^{2}}{2 \sigma_{2}^{2}}\right) .
\end{aligned}
$$

Let $s_{i}=0$. Then

$$
\frac{1}{2 \pi} h(0)=-\frac{p^{2}}{\sigma_{1}^{4}}-\frac{p(1-p)}{\sigma_{1} \sigma_{2}}\left(\frac{1}{\sigma_{1}^{2}}+\frac{1}{\sigma_{2}^{2}}\right)<0 .
$$

Now assume that $s_{i} \neq 0$. If we denote $t \triangleq\left(s_{i}^{2} / \sigma_{1}^{2}\right)$, then some terms in (38) are in the form of $t^{n} \exp (-t)$. If $\sigma_{1} \rightarrow 0$ or equivalently $t \rightarrow \infty$ then $t^{n} \exp (-t) \rightarrow 0$. Using these results, the limit of the function $h\left(s_{i}\right)$ is equal to

$$
\lim _{\sigma_{1} \rightarrow 0} h\left(s_{i}\right)=-\frac{(1-p)^{2}}{\sigma_{2}^{4}} \exp \left(\frac{-s_{i}^{2}}{\sigma_{2}^{2}}\right)<0
$$

which proves the concavity of $\log \left(p\left(s_{i}\right)\right)$. The convexity of $c(\mathbf{s})=(\mathbf{x}-\mathbf{A s})^{T}(\mathbf{x}-\mathbf{A} \mathbf{s})=\|\mathbf{x}-\mathbf{A s}\|^{2}$ is obvious due to its parabolic shape.

\section{B. Convergence of the Steepest-Ascent}

From (29) and by defining $\mathbf{s}_{k+1} \triangleq \mathbf{Q}_{k+1} \mathbf{r}_{k}, \mathbf{s}_{k} \triangleq \mathbf{Q}_{k} \mathbf{r}_{k}$, $\mathbf{H} \triangleq \mathbf{A}^{T} \mathbf{A}$ and $\mathbf{b}=2 \mathbf{x} \mathbf{A}^{T}$, we have

$$
\begin{aligned}
L\left(\mathbf{q}_{k+1}\right)- & L\left(\mathbf{q}_{k}\right)=\sum_{i=1}^{m} \log \frac{p\left(q_{k+1, i}\right)}{p\left(q_{k, i}\right)}-\frac{1}{2 \sigma_{n}^{2}} \\
& \times\left[\mathbf{s}_{k+1}^{T} \mathbf{H s}_{k+1}^{T}-\mathbf{s}_{k}^{T} \mathbf{H} \mathbf{s}_{k}^{T}-\mathbf{b}\left(\mathbf{s}_{k+1}-\mathbf{s}_{k}\right)\right] .
\end{aligned}
$$

If the above expression is positive, then the sequence $L\left(\mathbf{q}_{k}\right)$ is an increasing sequence and since $L(\mathbf{q})$ is upper bounded by the value $m \log (p)$, then the sequence has a limit and the convergence of the M-step (steepest-ascent) will be proved. To derive the positivity condition of (40), we write the M-step iteration as $\mathbf{q}_{k+1}=\mathbf{q}_{k}+\mu \mathbf{c}_{k}$ where $\mathbf{c}_{k}=\left.(\partial L(\mathbf{q}) / \partial \mathbf{q})\right|_{\mathbf{q}=\mathbf{q}_{k}}$. Therefore, we have $\mathbf{s}_{k+1}-\mathbf{s}_{k}=\mu \mathbf{C}_{k} \mathbf{r}_{k}$ where $\mathbf{C}_{k}=\operatorname{diag}\left(\mathbf{c}_{k}\right)$. Substituting in (40) results in

$$
\begin{aligned}
L\left(\mathbf{q}_{k+1}\right)- & L\left(\mathbf{q}_{k}\right)=\sum_{i=1}^{m} \log \frac{p\left(q_{k+1, i}\right)}{p\left(q_{k, i}\right)}-\frac{\mu}{2 \sigma_{n}^{2}} \\
& \times\left[\mu \mathbf{r}_{k}^{T} \mathbf{C}_{k} \mathbf{H} \mathbf{C}_{k} \mathbf{r}_{k}+\left(2 \mathbf{s}_{k}^{T} \mathbf{H}-\mathbf{b}\right) \mathbf{C}_{k} \mathbf{r}_{k}\right] .
\end{aligned}
$$

After some algebra detailed in Appendix III, one obtains the following inequality:

$$
\frac{1}{\mu}\left[L\left(\mathbf{q}_{k+1}\right)-L\left(\mathbf{q}_{k}\right)\right] \geqslant \mathbf{c}_{k}^{T}\left[\mathbf{I}-\mu \mathbf{R}-\frac{\mu}{2 \sigma_{n}^{2}} \mathbf{R}_{1} \mathbf{H} \mathbf{R}_{1}\right] \mathbf{c}_{k}
$$

where $\mathbf{R}_{1}=\operatorname{diag}\left(r_{i}\right)$ and $\mathbf{R}$ is defined in Appendix III. If the symmetric matrix $\mathbf{D} \triangleq \mathbf{I}-\mu \mathbf{R}-\left(\mu / 2 \sigma_{n}^{2}\right) \mathbf{R}_{1} \mathbf{H R}_{1}$ is Positive Definite (PD) then the sequence $L\left(\mathbf{q}_{k}\right)$ will be increasing, and, as mentioned above, the convergence of the M-step is guaranteed. For $\mathbf{D}$ being PD, all its eigenvalues should be positive. If we define $\mathbf{E} \triangleq \mathbf{R}+\left(1 / 2 \sigma_{n}^{2}\right) \mathbf{R}_{1} \mathbf{H R}_{1}$ then $\mathbf{D}=\mathbf{I}-\mu \mathbf{E}$. The PD property of $\mathbf{D}$ results in the following equation:

$$
\mu<\frac{1}{\lambda_{\max }(\mathbf{E})}
$$

where $\lambda_{\max }(\mathbf{E})$ stands for the maximum eigenvalue of $\mathbf{E}$. Since $\mathbf{R}$ is diagonal, $\lambda_{i}(\mathbf{E})=\lambda_{i}(\mathbf{R})+\left(1 / 2 \sigma_{n}^{2}\right) \lambda_{i}\left(\mathbf{R}_{1} \mathbf{H R}_{1}\right)$. Again since $\mathbf{R}$ is diagonal, its eigenvalues are $\lambda_{i}(\mathbf{R})=$ $\sum_{j=1}^{2}\left(r_{j}\left(q_{k, i}\right) / 2 \sigma_{0}^{2}\right)=\left(1 / 2 \sigma_{0}^{2}\right)$, then we have

$$
\lambda_{\max }(\mathbf{E})<\frac{1}{2 \sigma_{0}^{2}}+\frac{1}{2 \sigma_{n}^{2}} \lambda_{\max }\left(\mathbf{R}_{1} \mathbf{H R}_{1}\right) .
$$

To obtain an upper bound for the maximum eigenvalue of $\mathbf{E}$, we should find the upper bound for the maximum eigenvalue of $\mathbf{R}_{1} \mathbf{H R}_{1}$. Since $\mathbf{R}_{1} \mathbf{H} \mathbf{R}_{1}=(\mathbf{A R})^{T}\left(\mathbf{A R}_{1}\right)$, it is PD and hence all of its eigenvalues are positive. Therefore, we can write

$$
\lambda_{\max }\left(\mathbf{R}_{1} \mathbf{H} \mathbf{R}_{1}\right) \leqslant \sum_{i=1}^{m} \lambda_{i}=\operatorname{trace}\left(\mathbf{R}_{1} \mathbf{H} \mathbf{R}_{1}\right)=\operatorname{trace}\left(\mathbf{R}_{1}^{2} \mathbf{H}\right) .
$$

Since $\mathbf{R}_{1} \triangleq \operatorname{diag}\left(r_{i}\right)$, the elements of $\mathbf{R}_{1}$ and hence $\lambda_{\max }\left(\mathbf{R}_{1} \mathbf{H} \mathbf{R}_{1}\right)$ can theoretically be infinitely large. However, noting that $p\left(\left|r_{i}\right|<M\right)=1-2 Q\left(\left(M / \sigma_{r}\right)\right)$, we can determine a suitable interval for the maximum value of $\lambda_{\max }\left(\mathbf{R}_{1} \mathbf{H R}_{1}\right)$. For the cases which $\left|r_{i}\right|<M$, $\operatorname{trace}\left(\mathbf{R}_{1}^{2} \mathbf{H}\right)=\sum_{i=1}^{m} r_{i}^{2} h_{i i}<M^{2} \sum_{i=1}^{m} h_{i i}$ with probability $\gamma=\left(1-2 Q\left(\left(M / \sigma_{r}\right)\right)\right)^{m}$ which we want to be very close to 1 (for example $\gamma=0.99$ ). Hence, we must choose the value as $M^{*}=\sigma_{r} Q^{-1}\left(\frac{1-\sqrt[m]{\gamma}}{2}\right)$. Consequently, to be sure that with a probability $\gamma$ close to 1 the convergence is guaranteed, $\mu$ should be selected in the interval:

$$
0<\mu<\frac{2}{\frac{1}{\sigma_{0}^{2}}+\frac{M^{* 2}}{\sigma_{n}^{2}} \operatorname{trace}\left(\mathbf{A}^{T} \mathbf{A}\right)} .
$$

By imposing the condition of normalized column to $\mathbf{A}$, the diagonal elements of $\mathbf{A}^{T} \mathbf{A}$ is equal to one and hence we have $\operatorname{trace}\left(\mathbf{A}^{T} \mathbf{A}\right)=m$. Therefore, the suitable interval can be simplified as

$$
0<\mu<\frac{2}{\frac{1}{\sigma_{0}^{2}}+\frac{m M^{* 2}}{\sigma_{n}^{2}}} .
$$

\section{Convergence of the IBA Algorithm}

So far, we have shown the convergence of the M-step which is implemented with steepest-ascent method. To prove the convergence of the proposed IBA algorithm which is like an EM al- 
gorithm, we should prove that the sequence $L\left(\mathbf{s}_{k}\right)$ is increasing, where $L\left(\mathbf{s}_{k}\right)$ is the $\log$ posterior probability defined in (37). Increasing of this sequence is evident throughout the M-step because the $L(\mathbf{s})$ is equivalent to $L(\mathbf{q})$ throughout the M-step. In the E-step the LLS estimation of $\mathbf{r}$ is computed by (24). This estimation is also a MAP estimation. So, it maximizes our measure which is the posterior probability in the MAP sense. Since the logarithm function is monotonically increasing, MAP estimation is equivalent to maximizing the $\log$ posterior $L(\mathbf{s})$. So, the $\log$ posterior probability $L(\mathbf{s})$ increases in each E-step and $\mathrm{M}$-step, inside any $k$ th iteration. So, in each iteration we can write

$L\left(\mathbf{s}_{\mathrm{M}-\text { step }}^{(k+1)}\right)>L\left(\mathbf{s}_{\mathrm{E}-\text { step }}^{(k+1)}\right)>L\left(\mathbf{s}_{\mathrm{M}-\text { step }}^{(k)}\right)>L\left(\mathbf{s}_{\mathrm{E}-\text { step }}^{(k)}\right)$

where the sequences of sources are

$$
\begin{aligned}
\mathbf{s}_{\mathrm{E}-\text { step }}^{(k)} & =\mathbf{Q}_{k-1} \mathbf{r}_{k} \\
\mathbf{s}_{\mathrm{M}-\text { step }}^{(k)} & =\mathbf{Q}_{k} \mathbf{r}_{k} \\
\mathbf{s}_{\mathrm{E}-\text { step }}^{(k+1)} & =\mathbf{Q}_{k} \mathbf{r}_{k+1} \\
\mathbf{s}_{\mathrm{M}-\text { step }}^{(k+1)} & =\mathbf{Q}_{k+1} \mathbf{r}_{k+1} .
\end{aligned}
$$

Therefore, the sequence of $L\left(\mathbf{s}_{k}\right)$ is converging to a local maximum $\mathbf{s}^{*}$. The concavity of the $L(\mathbf{s})$ guarantee that this local maximum is equal to the global maximum which is the MAP solution of sparse sources.

\section{EXPERIMENTAL RESULTS}

In this section, the performance of the MAP algorithm and IBA algorithm is examined with concentrating on the IBA algorithm. The results of our algorithms are compared to the BP which is practically implemented by $\ell^{1}$-magic [50] and some other algorithms which are in the literature. This will be done by discussing the results of five experiments detailing different aspects of our algorithms, especially the effects of the parameters, of noise and of sparsity.

The sparse sources are artificially generated using the BG model in (2). In all simulations, we used $p=0.9, \sigma_{n}=0.01$ and $\sigma_{r}=1$ for the parameters of the BG model. A different source model will be used in the fourth experiment for which the number of active sources is fixed and the locations of the active sources are chosen randomly. The noise vector is an additive white Gaussian noise with covariance matrix $\sigma_{n}^{2} \mathbf{I}$. For investigating the noise effect, we define an input signal-to-noise ratio as

$$
\text { Input-SNR } \triangleq 20 \log \left(\frac{\sigma_{r}}{\sigma_{n}}\right) .
$$

The mixing matrix entries are chosen randomly from a uniform distribution in $[-1,1]$ and then the columns of the mixing matrix are normalized to unity. To evaluate the performance of our algorithms, we use two definitions. In sparse decomposition experiments (single realization of $\mathbf{x}=\mathbf{A s}+\mathbf{n}$ ), the output signal-to-noise ratio defined as

$$
\text { Output-SNR }=20 \log \left(\|\mathbf{s}\|_{2} /\|\mathbf{s}-\widehat{\mathbf{s}}\|_{2}\right)
$$

is used as the performance measure. But in sparse component analysis experiments (many realizations of $\{\mathbf{x}(t)=\mathbf{A} \mathbf{s}(t)+$ $\mathbf{n}(t)\}_{t=1}^{N}$ ), we usually average over time to obtain the Individual $S N R$ for each source, defined by

$$
\mathrm{SNR}_{i}=10 \log \left(\frac{\sum_{t=1}^{N} s_{i}^{2}(t)}{\sum_{t=1}^{N}\left(s_{i}(t)-\widehat{s}_{i}(t)\right)^{2}}\right)
$$

and the final performance index (SNR) is the average of these SNRs.

For each experiment, the performance indexes are computed by averaging over 400 different realizations with new randomly chosen mixing matrix and sparse sources.

We use the CPU time as a measure of complexity. Although, the CPU time is not an exact measure, it can give us a rough estimation of the complexity for comparing our algorithm with other algorithms. Our simulations were performed in MATLAB7.0 environment using an Intel 2.80-GHz processor with 1024 MB of RAM and under Linux operating system.

\section{A. Experiment 1-Performance Evaluation of the MAP Algorithm}

1) Parameter Estimation: In this experiment, the main goal is to investigate the parameter estimation techniques described in Section III-A. Eight sources $(m=8)$ are selected with source parameters $p=.9, \sigma_{r}=1$ and the noise parameter $\sigma_{n}=.01$. Then, the mixture signals are obtained by the model (1). This experiment estimates the three unknown parameters from only $N$ observations (or samples) of mixtures. Furthermore, 100 iterations are used for the EM algorithm for estimating $\sigma_{n}$. To measure the performance of estimating these parameters, we computed the normalized mean square error, defined as follows. Let

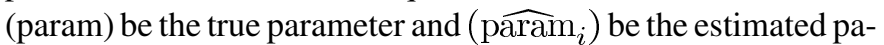
rameter in the $i$ th experiment, then the normalized mean square error (over the 400 realizations) is defined as

$$
e_{n}=\frac{\frac{1}{400} \sum_{i=1}^{400}\left(\text { param }^{2} \text { param }_{i}\right)^{2}}{\text { param }^{2}} .
$$

The normalized mean square errors (in percent) versus the observation number are depicted in Fig. 3. As it can be seen, even for small number of samples (due to sparsity, the significant samples are then very few) the estimates for $p, \sigma_{r}$ are acceptable. By increasing the number of observations, better estimates of parameters are obtained and the errors decrease.

2) Overall Source Estimation: In this experiment, the MAP algorithm is compared to BP. Moreover, to investigate the effect of parameter estimation error, the MAP algorithm was simulated in two cases: one with the actual parameters and the other with estimated parameters.

As before, the simulation parameters are $m=8, p=.9$, $\sigma_{r}=1$ and $\sigma_{n}=.01$. The number of samples is $N=1000$ and the number of mixtures $(n)$ varies between 3 to 7 . The results of three algorithms, namely BP, MAP with actual parameters and MAP with estimated parameters (all of them averaged on 400 simulations) are shown in Fig. 4. The results show that the average SNR (Temporal SNR) of the MAP algorithm is about $10 \mathrm{~dB}$ better than BP. Moreover, the averaged SNR of the MAP algorithm with estimated parameters is close to the averaged 


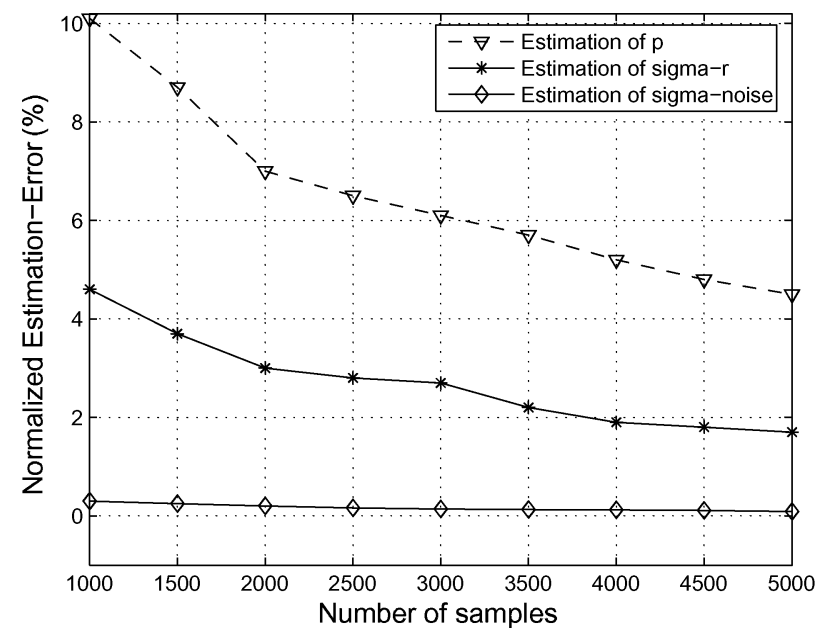

Fig. 3. The result of parameter estimation of the MAP algorithm in the case where $m=8, n=4, p=.9, \sigma_{r}=1$ and $\sigma_{n}=.01$. The results are averaged on 400 simulations.

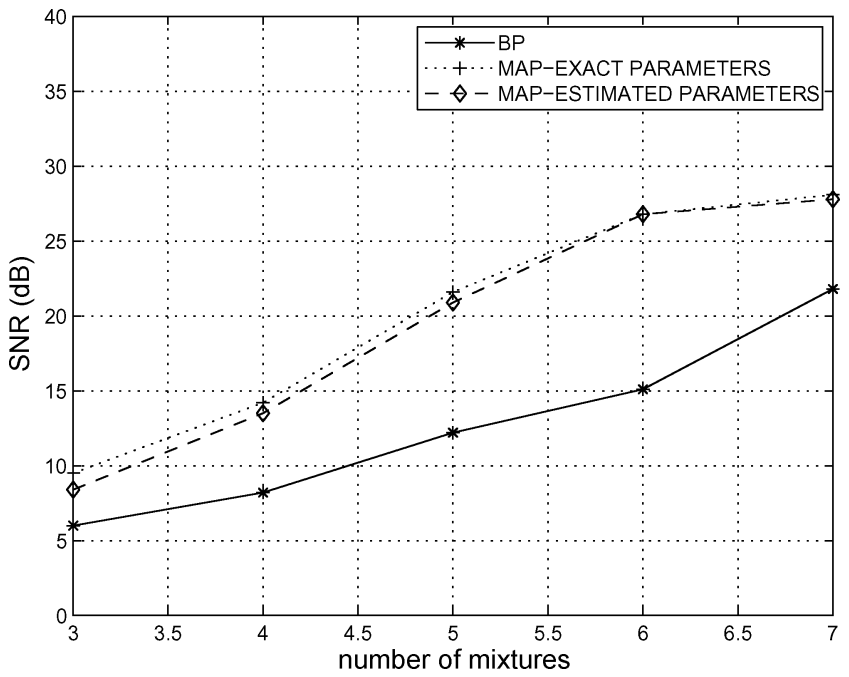

Fig. 4. The performance of MAP algorithm (with actual parameters and with estimated parameters) in comparison to LP method in the presence of noise, where $m=8, N=1000, p=.9, \sigma_{r}=1$ and $\sigma_{n}=.01$. The results are averaged on 400 simulations.

SNR with actual parameters. Consequently, the MAP algorithm is robust to the parameter errors: in fact, for $N=1000$, although the relative estimation errors of $p$ and $\sigma_{r}$ achieve about $10 \%$ and $5 \%$ (Fig. 3), the SNR loss with estimated parameters is less than $1 \mathrm{~dB}$ (Fig. 4).

\section{B. Experiment 2-Performance Evaluation of the IBA Algorithm}

In this section, the performance of the IBA algorithm is investigated, and compared with different algorithms for various noise levels i.e., input SNRs. In this experiment, the simulation parameters are $m=1024, n=512, p=.9, \sigma_{n}=0.01$ and $\sigma_{r}=1$. In the M-step the value of $\alpha$ can be chosen between 0.6 and 0.9 (see the third experiment which investigates the parameter selection). In this experiment, we select $\alpha=0.8$. The initial value of $\sigma_{0}$ is equal to 1 . In this special case the suitable interval of $\mu$ in (32) is $0<\mu<2.1466 \times 10^{-7}$. So, we
TABLE I

Progress OF the ItERATIVE PARAMETER ESTIMATION to ACtUAL PARAMETERS IN THE CASE OF $m=1024, n=512$ AND $p=0.9 \sigma_{r}=1 \sigma_{n}=.01$

\begin{tabular}{c|c|c|c}
\hline itr. \# & $p$ & $\sigma_{r}$ & $\sigma_{n}$ \\
\hline 1 & 0.8000 & 0.7412 & 0.0782 \\
2 & 0.8400 & 0.8310 & 0.0524 \\
3 & 0.9070 & 1.0412 & 0.0082 \\
4 & 0.9119 & 1.1206 & 0.0048 \\
5 & 0.9119 & 1.1206 & 0.0055 \\
\hline
\end{tabular}

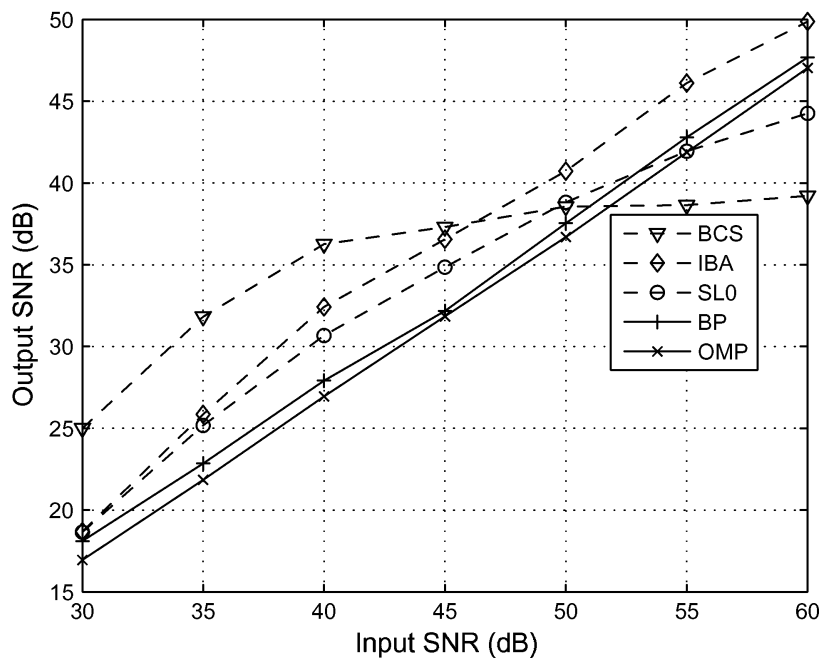

Fig. 5. Output-SNR versus Input-SNR. Results of the IBA algorithm compared with other algorithms. The simulation parameters are $m=1024, n=512$, $p=.9, \sigma_{r}=1, \alpha=.8$, and $\mu=10^{-7}$. Five iterations are used for the M-step (steepest-ascent). Results are averaged on 400 simulations.

chose $\mu=10^{-7}$. For the initial value of the parameter $p$, we selected the value $\hat{p}^{(0)}=0.8$ because it is a good tradeoff, and the initial value must imply a sufficient number of active sources. The practical value of $T h$ in Fig. 2, is selected as $\hat{\sigma}_{r}^{(0)} / 4$ because it provides slightly better results (refer to experiment 3 ). The simulations show that, for these parameters, only 4 or 5 iterations are sufficient to maximize the expression $L(\mathbf{q})$ in the M-step. IBA algorithm usually converges at the third and fourth iterations in our simulations. Therefore, 5 iterations are used for the M-step and for the overall IBA algorithm, we stop the algorithm when the condition $\left(\left\|\mathbf{s}^{(k)}-\mathbf{s}^{(k-1)}\right\|_{2} /\left\|\mathbf{s}^{(k)}\right\|_{2}\right)<0.001$ is satisfied.

Table I shows the progress of iterative parameter estimation to actual parameters. The results of output SNR versus input SNR are shown in Fig. 5 for our IBA algorithms and some other algorithms.

For comparison, we selected 4 representative algorithms. The first algorithm is OMP which is a pursuit algorithm which uses correlations between the signal and the atoms for selecting the current active atom. It also projects the residual signal to the surface of active atoms to update the coefficients at the end of each iteration. The second algorithm is BP which replaces $\ell^{0}$-norm by $\ell^{1}$-norm for the sparsity measure and uses LP methods. The third algorithm is smoothed- $\ell^{0}$ (SL0) which replaces the $\ell^{0}$-norm by a smoothed approximation of $\ell^{0}$-norm and then uses a steepest-descent method to find the sparse coefficient. Finally, the fourth algorithm is BCS algorithm which 


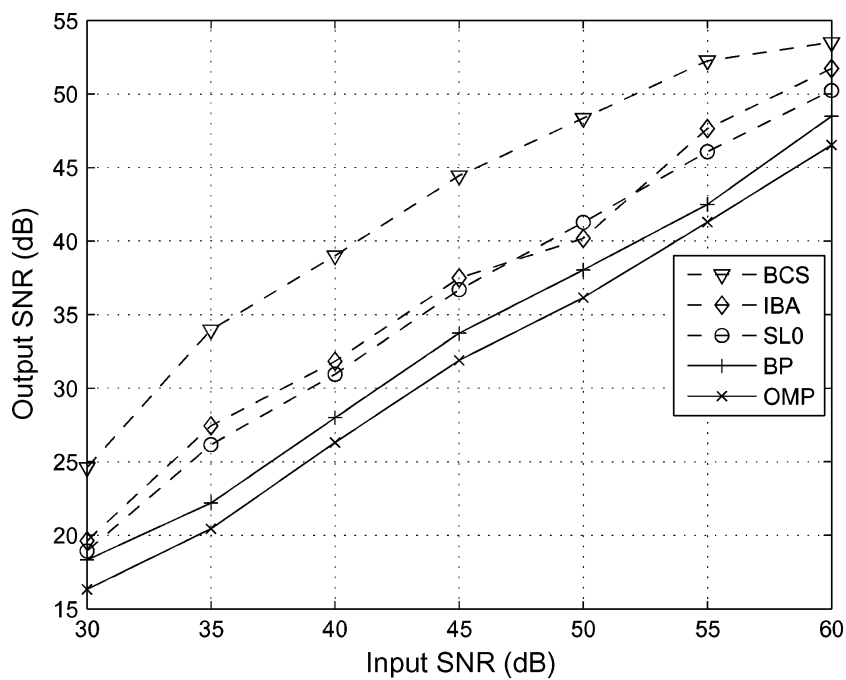

Fig. 6. Results of the IBA algorithm compared with other algorithms, for non BG sources. The simulation parameters are $m=1024, n=512, p=.9$, $\sigma_{r}=1, \alpha=.8$, and $\mu=10^{-7}$. Five iterations are used for the M-step (steepest-ascent). Results are averaged on 400 simulations.

is a Bayesian algorithm which uses a hierarchical sparseness prior and a type-II maximum-likelihood (ML) procedure like the RVM method to estimate the unknown variables.

The number of iterations are 100 for OMP. For smoothed- $\ell^{0}$, we used a sequence of $\sigma$ as $\sigma^{(k)}=0.9 \sigma^{(k-1)}$ from the initial estimated value to the final value 0.04 and $L=3$ and $\mu=2$ [25]. For BCS and BP, we used the author recommendations for choosing the parameters. As we can see from the figure, our performance is somewhat better than other methods like BP, OMP and SL0. Only, BCS provides better performance specially for low SNRs.

We ran another experiment for sources, different of the BG model. In this experiment, the sources are fixed and equal to 1 from index 1 to 20 and also are fixed and equal to 0 for other indexes (from 21 to 1024). The results for this special case are shown in Fig. 6. In this special case, the IBA algorithm achieves the second best performance, just after BCS algorithm.

\section{Experiment 3-Dependence on the Parameters}

In this experiment, the effect of the simulation parameters $(\alpha$ and $T h$ in Fig. 2) is investigated.

1) Effect of $\alpha$ : In this experiment, we consider the effect of the parameter $\alpha$, which is the scale factor controlling the decreasing rate of $\sigma_{0}\left(\sigma_{0}^{(i)}=\alpha \sigma_{0}^{(i-1)}\right)$. The experiment results are shown in Fig. 7, which represents the averaged output-SNR versus $\alpha$, for different values of $\sigma_{n}$ [Fig. 7(a)] and $k=m(1-p)$ [Fig. 7(b)]. The value $\sigma_{n}$ determines the noise standard deviation. The value $k=m(1-p)$, which represents the average number of active sources, determines the degree of sparsity of the sources.

It is clear from Fig. 7(a) that the value of $\alpha$ can be selected properly between a lower bound (0.6) and an upper bound (0.9). In this experiment the values of other parameters are $\mu=10^{-7}$, $k=m(1-p)=100$. The performance decreases when $\alpha$ is too close to one (in this case .95). However, our experiments show the low sensitivity of our algorithm to this parameter. A good choice of this parameter is around 0.8 .

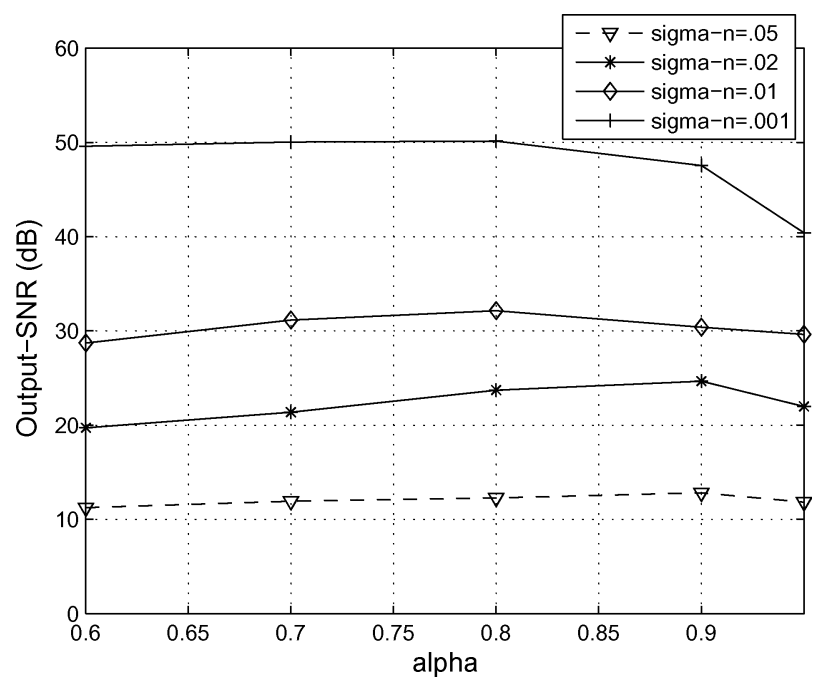

(a)

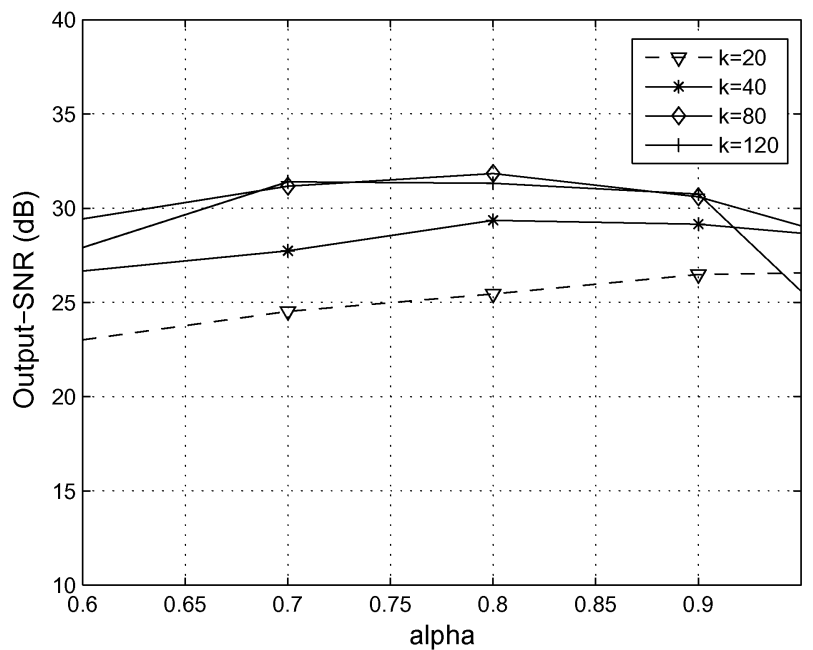

(b)

Fig. 7. Performance of the IBA algorithm versus parameter $\alpha$ for $m=1024$, $n=512$ and $\sigma_{r}=1$. In (a) $k$ (number of active sources) is fixed to 100 and the effect of noise is investigated. In (b) $\sigma_{n}$ is fixed to 0.01 and the effect of sparsity factor is analyzed. Values of $k$ are 20,40,80,120. Results are averaged on 400 simulations.

From [1] and [2], we know that $k \leq n / 2$ is a theoretical limit for uniqueness of the sparsest solution. In Fig. 7(b), the results of the SNR versus $\alpha$ in various sparsity conditions $(20 \leq k \leq 120)$ are shown. Again, our experiments show the IBA algorithm is not too sensitive to the parameter $\alpha: \alpha=0.8$ is still a good choice.

2) Effect of Th: In this experiment, the effect of the threshold $T h$ in Fig. 2 is investigated. We define a threshold factor as

$$
\text { Threshold-factor } \triangleq \frac{\hat{\sigma_{r}}}{T h} \text {. }
$$

We ran the IBA algorithm with various values of this threshold factor. The results are shown in Fig. 8 which represents the output-SNR versus the threshold factor. Although the algorithm has a low sensitivity to this threshold, but the value for the threshold factor should be less than 5. Arbitrarily, we chose a threshold factor equal to 4 , and $T h=\hat{\sigma_{r}} / 4$ in the IBA algorithm. 


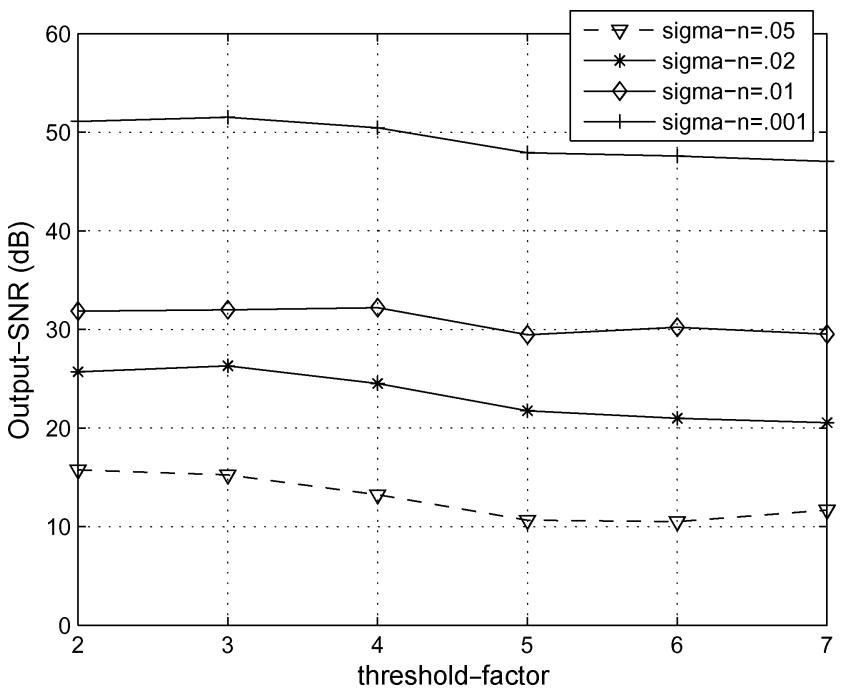

Fig. 8. Effect of the threshold in the IBA algorithm. The simulation parameters are $m=1024, n=512, p=.9, \sigma_{r}=1$ and $\mu=10^{-7}$. Results are averaged on 400 simulations.

\section{Experiment 4-Effect of Sparsity on the Performance}

Here, we experimentally consider the question: How much sparse a source should be to make the decomposition possible? As mentioned before, we have the theoretical limit of $n / 2$ on the maximum number of active sources at each sample (column) of source matrix to insure the uniqueness of the sparsest solution. But most of SCA methods are unable to practically achieve this limit [1].

To be able to measure the effect of sparsity, instead of generating the sources according to BG model (2), at each time $t$, we activate exactly $k$ entries out of $m$ components of $\mathbf{s}(t)$, (the column $t$ of the source matrix). The locations and the values of these $k$ elements are chosen randomly. $k$ is related to the sparsity level of the sources, defined as

$$
\text { Sparsity-level } \triangleq \frac{k}{n}
$$

where $k$ is the number of active sources.

Fig. 9 shows the output SNR (averaged on 400 simulations), as a function of sparsity level, for several values of $\alpha$ and compares the results with other algorithms. It can be seen that the IBA algorithm achieves a good performance especially when the sparsity level is low.

\section{E. Experiment 5-Complexity}

In this last experiment, the relative complexity (or speed) of the IBA algorithm is compared to other algorithms. The measure to be used is the "average CPU time" required by each algorithm. More specifically, we plot "average time" versus $m$, where $m$ is the number of sources. The number of mixtures is $n=0.5 \mathrm{~m}$. The various values of $m$ are 128, 256, 512, and 1024 . Fig. 10 summarizes the results of average simulation times (on 400 simulation) for recovering the sources at one time step. As we can see, the IBA algorithm has relatively a high complexity, a price paid for its good performance.

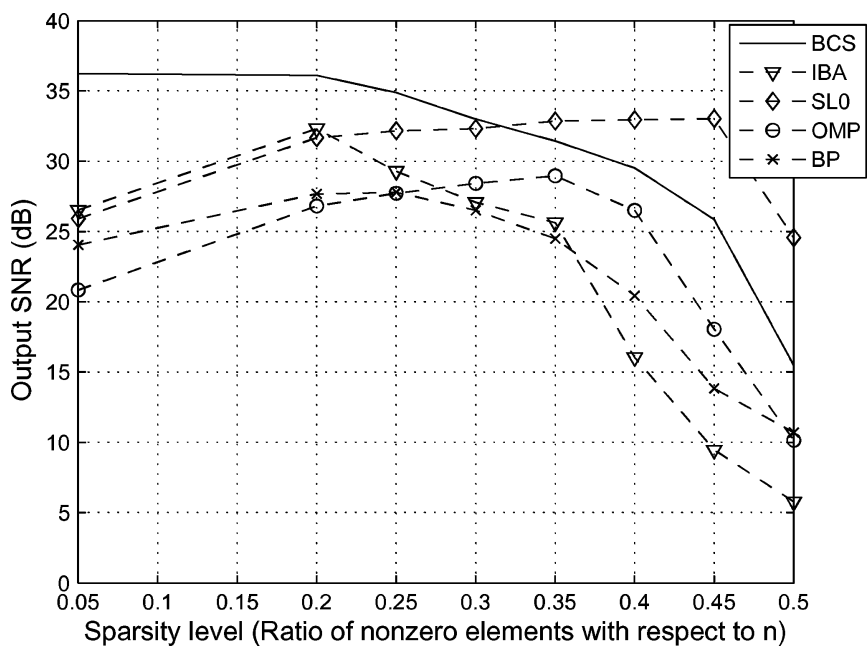

Fig. 9. Output-SNR as a function of sparsity level (active sources $/ n$ ) for various algorithms. Simulation parameters are $m=1024, n=512, \sigma_{r}=1$ and $\sigma_{n}=0.01$. Results are averaged on 400 simulations.

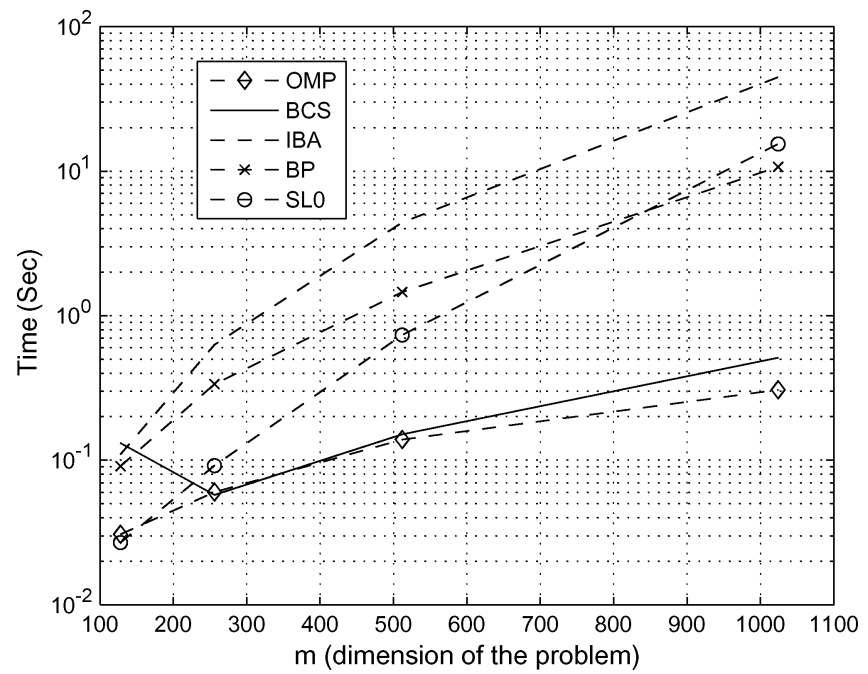

Fig. 10. Average CPU time in seconds versus problem dimension $(m)$. For all dimensions $n=0.5 \mathrm{~m}$. The parameters are $p=.9, \sigma_{r}=1$ and $\sigma_{n}=.01$. The results are averaged on 400 simulations.

\section{CONCLUSION}

In this paper, we have presented a new IBA algorithm for finding sparse solutions of underdetermined system of linear equations in the context of SCA. This IBA algorithm is based on iterative MAP estimation of the sources. In this paper, the high complexity of Bayesian methods is reduced by an iterative algorithm which resembles the EM algorithm. The M-step is done by a steepest-ascent method. Its step size should be chosen properly for insuring convergence of the steepest-ascent and of the global IBA algorithm. Moreover, the paper includes the convergence analysis of the algorithm and the convergence proof to the true optimum solution. Experimental results show the IBA algorithm has a low sensitivity to the simulation parameters, and achieves good performance, although not the best, compared with other algorithms especially BCS, which is currently the best algorithm, both for accuracy and speed. Future works includes improvement of Bayesian algorithms, by explicitly taking into account the noise for achieving performance as optimum as possible. 


\section{APPENDIX I}

DERIVATION OF THE STEEPEST-ASCENT

From (28), we have

$\frac{\partial L(\mathbf{q})}{\partial \mathbf{q}}=\frac{\partial}{\partial \mathbf{q}} \sum_{i=1}^{m} \log \left(p\left(q_{i}\right)\right)-\frac{1}{2 \sigma_{n}^{2}} \frac{\partial}{\partial \mathbf{q}}(\mathbf{x}-\mathbf{A} \mathbf{Q} \widehat{\mathbf{r}})^{T}(\mathbf{x}-\mathbf{A} \mathbf{Q} \widehat{\mathbf{r}})$.

We define $\mathbf{g}(\mathbf{q}) \triangleq-\sigma_{0}^{2}(\partial / \partial \mathbf{q}) \sum_{i=1}^{m} \log \left(p\left(q_{i}\right)\right)$ and $\mathbf{n}(\mathbf{q}) \triangleq$ $(\mathbf{x}-\mathbf{A Q} \widehat{\mathbf{r}})^{T}(\mathbf{x}-\mathbf{A Q} \widehat{\mathbf{r}})$. With these definitions the scalar function $g\left(q_{i}\right)$ and the $\mathbf{n}(\mathbf{q})$ (with omitting the constant terms) can be computed as

$$
\begin{aligned}
& g\left(q_{i}\right)=\frac{p q_{i} \exp \left(\frac{-q_{i}^{2}}{2 \sigma_{0}^{2}}\right)+(1-p)\left(q_{i}-1\right) \exp \left(\frac{-\left(q_{i}-1\right)^{2}}{2 \sigma_{0}^{2}}\right)}{p \exp \left(\frac{-q_{i}^{2}}{2 \sigma_{0}^{2}}\right)+(1-p) \exp \left(\frac{-\left(q_{i}-1\right)^{2}}{2 \sigma_{0}^{2}}\right)} \\
& \mathbf{n}(\mathbf{q})=-2 \mathbf{x}^{T} \mathbf{A} \mathbf{Q} \widehat{\mathbf{r}}+\widehat{\mathbf{r}}^{T} \mathbf{Q} \mathbf{A}^{T} \mathbf{A} \mathbf{Q} \widehat{\mathbf{r}} .
\end{aligned}
$$

With the definitions $\mathbf{C} \triangleq \mathbf{A}^{T} \mathbf{A}, \mathbf{n}_{\mathbf{1}}(\mathbf{q}) \triangleq-2 \mathbf{x}^{T} \mathbf{A Q} \widehat{\mathbf{r}}$ and $\mathbf{n}_{2}(\mathbf{q}) \triangleq \widehat{\mathbf{r}}^{T} \mathbf{Q C Q} \widehat{\mathbf{r}}$, we can write

$$
\frac{\partial n_{1}(\mathbf{q})}{\partial \mathbf{q}}=\operatorname{diag}\left(-2 \mathbf{x}^{T} \mathbf{A}\right) \cdot \widehat{\mathbf{r}}
$$

If we define $\mathbf{W} \triangleq \mathbf{Q} \widehat{\mathbf{r}}(m \times 1$ vector $)$ then $\mathbf{n}_{2}(\mathbf{q})=\mathbf{W}^{T} \mathbf{C W}$ and so we have

$$
\frac{\partial \mathbf{n}_{2}(\mathbf{q})}{\partial q_{i}}=\sum_{j=1}^{m} \frac{\partial \mathbf{n}_{2}(\mathbf{q})}{\partial W_{j}} \frac{\partial W_{j}}{\partial q_{i}} .
$$

From the vector derivatives, we have $\left(\partial \mathbf{n}_{2}(\mathbf{q}) / \partial \mathbf{W}\right)=$ $2 \mathbf{C W} \triangleq \mathbf{d}$. And from the definition of $\mathbf{W}$ we get $\left(\partial W_{j} / \partial q_{i}\right)=\widehat{r}_{i} \delta_{i j}$. So (61) is converted to $\left(\partial \mathbf{n}_{2}(\mathbf{q}) / \partial q_{i}\right)=$ $\sum_{j=1}^{m} d_{j} \widehat{r}_{i} \delta_{i j}=\widehat{r}_{i} d_{i}$. So the vector form of (61) is equal to

$$
\frac{\partial \mathbf{n}_{2}(\mathbf{q})}{\partial \mathbf{q}}=\operatorname{diag}(\mathbf{d}) . \hat{\mathbf{r}} \text {. }
$$

From (60), (62), $\mathbf{n}(\mathbf{q})=\mathbf{n}_{1}(\mathbf{q})+\mathbf{n}_{2}(\mathbf{q})$ and definitions of vectors $\mathbf{d}$ and $\mathbf{C}$, we can write

$$
\frac{\partial \mathbf{n}(\mathbf{q})}{\partial \mathbf{q}}=2 \operatorname{diag}\left(\mathbf{A}^{T} \mathbf{A} \mathbf{Q} \widehat{\mathbf{r}}-\mathbf{A}^{T} \mathbf{x}\right) \cdot \widehat{\mathbf{r}} .
$$

Finally, (63), (57), (30) with the definitions of $\mathbf{n}(\mathbf{q})$ and $\mathbf{g}(\mathbf{q})$ yields the steepest-ascent iteration (31).

\section{APPENDIX II}

\section{MAP ESTIMATION OF PARAMETERS}

To compute the MAP estimate of $\sigma_{r}$, assuming the other parameters are known, we should maximize the posterior probability $p\left(\sigma_{r} \mid \hat{\mathbf{r}}, \hat{\mathbf{q}}, \hat{\sigma_{n}}, \hat{p}, \mathbf{x}\right) \equiv p\left(\sigma_{r}\right) p\left(\hat{\mathbf{r}} \mid \sigma_{r}\right)$. We do not impose any prior information about $\sigma_{r}$. So, the MAP estimation of $\sigma_{r}$ should maximize $p\left(\hat{\mathbf{r}} \mid \sigma_{r}\right)$. This distribution is equal to

$$
p\left(\hat{\mathbf{r}} \mid \sigma_{r}\right)=\left(2 \pi \sigma_{r}^{2}\right)^{-\frac{m}{2}} \exp \left(\frac{-1}{2 \sigma_{r}^{2}} \hat{\mathbf{r}}^{T} \hat{\mathbf{r}}\right) .
$$

Differentiating the above equation with respect to $\sigma_{r}$, the MAP estimate of $\sigma_{r}$ is

$$
\hat{\sigma}_{r, \mathrm{MAP}}^{2}=\frac{1}{m}\|\mathbf{r}\|_{2}^{2}
$$

To compute the MAP estimate of $\sigma_{n}$, assuming the other parameters are known, we must maximize the posterior probability $p\left(\sigma_{n} \mid \hat{\mathbf{q}}, \hat{\mathbf{r}}, \hat{\sigma_{r}}, \hat{p}, \mathbf{x}\right) \equiv p\left(\mathbf{x} \mid \sigma_{n}, \hat{\mathbf{r}}, \hat{\mathbf{q}}\right)=p_{n}(\mathbf{x}-\mathbf{A} \hat{\mathbf{Q}} \hat{\mathbf{r}})$. This probability distribution is equal to

$p_{n}(\mathbf{x}-\mathbf{A} \hat{\mathbf{Q}} \hat{\mathbf{r}})=\left(2 \pi \sigma_{n}^{2}\right)^{-\frac{n}{2}} \exp \left(\frac{-1}{2 \sigma_{n}^{2}}(\mathbf{x}-\mathbf{A} \hat{\mathbf{Q}} \hat{\mathbf{r}})^{T}(\mathbf{x}-\mathbf{A} \hat{\mathbf{Q}} \hat{\mathbf{r}})\right)$.

Differentiating the above equation with respect to $\sigma_{n}$ yields to the following equation:

$$
\hat{\sigma}_{n, \mathrm{MAP}}^{2}=\frac{\|\mathbf{x}-\mathbf{A} \hat{\mathbf{s}}\|_{2}^{2}}{n} .
$$

Finally, to calculate the MAP estimate of $p$, assuming the other parameters are known, the posterior probability $p\left(p \mid \hat{\mathbf{q}}, \hat{\mathbf{r}}, \hat{\sigma_{r}}, \hat{\sigma_{n}}, \mathbf{x}\right)$ should be maximized. This probability is equivalent to $p(\hat{\mathbf{q}} \mid p) p\left(\mathbf{x} \mid \hat{\mathbf{q}}, \hat{\mathbf{r}}, \hat{\sigma_{n}}\right)$ in which only the term $p(\hat{\mathbf{q}} \mid p)$ depends on $p$. Differentiating $p(\hat{\mathbf{q}} \mid p)=p^{\left(m-n_{a}\right)}(1-p)^{n_{a}}$ with respect to $p$ and setting it to zero, results in the following estimate of $p$ :

$$
\hat{p}_{\mathrm{MAP}}=\frac{m-n_{a}}{m}=\frac{\|\mathbf{q}\|_{0}}{m} .
$$

\section{APPENDIX III}

\section{DERIVING INEQUALITY}

For calculating the first term of (41) $B \triangleq$ $\sum_{i=1}^{m} \log \left(p\left(q_{k+1, i}\right) / p\left(q_{k, i}\right)\right)$, we define $p\left(q_{i}\right) \triangleq$ $\sum_{j=1}^{2} \pi_{j} g_{j}\left(q_{i}\right)$ where $\pi_{1} \triangleq p$ and $\pi_{2} \triangleq 1-p$ and $g_{j}\left(q_{i}\right)=\left(1 / \sigma_{0} \sqrt{2 \pi}\right) \exp \left(\left(-\left(q_{i}-m_{j}\right)^{2} / 2 \sigma_{0}^{2}\right)\right)$. Based on (22), we selected the two variances equal to $\sigma_{0}$ and $m_{1}=0$ and $m_{2}=1$. Then we will have

$$
\begin{aligned}
& B=\sum_{i=1}^{m} \log \left\{\frac{\sum_{j} \pi_{j} g_{j}\left(q_{k, i}+\mu c_{k, i}\right)}{\sum_{j} \pi_{j} g_{j}\left(q_{k, i}\right)}\right\} \\
& B=\sum_{i=1}^{m} \log \left\{\frac{\sum_{j} \pi_{j} g_{j}\left(q_{k, i}\right) \exp \left(\frac{-\mu^{2} c_{k, i}^{2}-2 \mu c_{k, i}\left(q_{k, i}-m_{j}\right)}{2 \sigma_{0}^{2}}\right)}{\sum_{j} \pi_{j} g_{j}\left(q_{k, i}\right)}\right\}
\end{aligned}
$$

where $c_{k, i}$ is $i$ th element of $\mathbf{c}_{k}$. By defining $r_{j}\left(q_{k, i}\right) \triangleq$ $\left(\pi_{j} g_{j}\left(q_{k, i}\right) / \sum_{j} \pi_{j} g_{j}\left(q_{k, i}\right)\right)$ (note that $0<r_{j}\left(q_{k, i}\right)<1$ ) and by considering the concavity of the logarithm function, we can write

$$
\begin{aligned}
B=\sum_{i=1}^{m} \log \{ & \sum_{j=1}^{2} r_{j}\left(q_{k, i}\right) \\
& \left.\quad \times \exp \left(\frac{-\mu^{2} c_{k, i}^{2}-2 \mu c_{k, i}\left(q_{k, i}-m_{j}\right)}{2 \sigma_{0}^{2}}\right)\right\} \\
\geqslant & \sum_{i=1}^{m} \sum_{j=1}^{2} \frac{r_{j}\left(q_{k, i}\right)}{2 \sigma_{0}^{2}}\left[-\mu^{2} c_{k, i}^{2}-2 \mu c_{k, i}\left(q_{k, i}-m_{j}\right)\right] .
\end{aligned}
$$

After some simplifications, the lower bound of $B$ in (70) becomes

$$
C \triangleq \sum_{i=1}^{m}-\mu^{2} c_{k, i}^{2} \frac{\gamma_{k, i}}{2 q_{k, i}}+\sum_{i=1}^{m}-\mu c_{k, i} \gamma_{k, i}+\sum_{i=1}^{m} \mu c_{k, i} \frac{r_{2}\left(q_{k, i}\right)}{\sigma_{0}^{2}}
$$


where $\gamma_{k, i} \triangleq q_{k, i} \sum_{j=1}^{2}\left(r_{j}\left(q_{k, i}\right) / \sigma_{0}^{2}\right)$. We know that $\mathbf{c}=(\partial L(\mathbf{q}) / \partial \mathbf{q})$. So we have

$c_{i}=\frac{-1}{2 \sigma_{n}^{2}} \frac{\partial}{\partial q_{i}}(\mathbf{x}-\mathbf{A s})^{T}(\mathbf{x}-\mathbf{A} \mathbf{s})+\frac{\partial}{\partial q_{i}}\left(\sum_{i=1}^{m} \log \sum_{j=1}^{2} \Pi_{j} p_{j}\left(q_{i}\right)\right)$

where $c_{i}$ is the $i$ th element of $\mathbf{c}$. If we define $N \triangleq(\mathbf{x}-$ $\mathbf{A s})^{T}(\mathbf{x}-\mathbf{A s})$ and $M=\sum_{i=1}^{m} \log \sum_{j=1}^{2} \pi_{j} g_{j}\left(q_{i}\right)$, then we can write $\left(\partial N / \partial q_{i}\right)=\left(\partial N / \partial s_{i}\right)\left(\partial s_{i} / \partial q_{i}\right)=\left[2 \mathbf{A}^{T}(\mathbf{A} \mathbf{s}-\mathbf{x})\right]_{i} r_{i}$. Moreover, we have

$$
\frac{\partial M}{\partial q_{i}}=\frac{\pi_{1} \frac{d}{d q_{i}} g_{1}\left(q_{i}\right)+\pi_{2} \frac{d}{d q_{i}} g_{2}\left(q_{i}\right)}{\sum_{j=1}^{2} \pi_{j} g_{j}\left(q_{i}\right)} .
$$

After some simplifications, we have

$$
\frac{\partial M}{\partial q_{i}}=-q_{i} \sum_{j=1}^{2} \frac{r_{j}\left(q_{i}\right)}{\sigma_{0}^{2}}+\frac{r_{2}\left(q_{i}\right)}{\sigma_{0}^{2}}
$$

Therefore, we have

$$
\mathbf{c}_{k}=-\mathbf{z}_{k}-\boldsymbol{\gamma}_{k}+\frac{\mathbf{r}_{2}\left(\mathbf{q}_{k}\right)}{\sigma_{0}^{2}}
$$

where $\mathbf{r}_{2}(\mathbf{q})=\left[r_{2}\left(q_{1}\right), r_{2}\left(q_{2}\right), \ldots, r_{2}\left(q_{m}\right)\right]^{T}$ and $r_{2}\left(q_{i}\right) \triangleq$ $\left(\pi_{2} g_{2}\left(q_{i}\right) / \sum_{j} \pi_{2} g_{2}\left(q_{i}\right)\right)$ and $z_{i} \triangleq\left(1 / 2 \sigma_{n}^{2}\right)\left[2 \mathbf{A}^{T}(\mathbf{A s}-\mathbf{x})\right]_{i} r_{i}$ and $\gamma_{i} \triangleq q_{i} \sum_{j=1}^{2}\left(r_{j}\left(q_{i}\right) / \sigma_{0}^{2}\right)$. Finally, the lower bound $C$ in (71) can be simplified as

$$
C=-\mu^{2} \mathbf{c}_{k}^{T} \mathbf{R} \mathbf{c}_{k}-\mu \mathbf{c}_{k}^{T} \gamma_{k}+\frac{\mu}{\sigma_{0}^{2}} \mathbf{c}_{k}^{T} \mathbf{r}_{2}\left(\mathbf{q}_{k}\right)
$$

where $\mathbf{R} \triangleq \operatorname{diag}\left(\sum_{j=1}^{2}\left(r_{j}\left(q_{k, i}\right) / 2 \sigma_{0}^{2}\right)\right)=\operatorname{diag}\left(\gamma_{k, i} / 2 q_{k, i}\right)$. From (41), (69), (70), (71), and (76), we have

$$
\begin{gathered}
\boldsymbol{L}\left(\mathbf{q}_{k+1}\right)- \\
-L\left(\mathbf{q}_{k}\right) \geqslant-\mu^{2} \mathbf{c}_{k}^{T} \mathbf{R} \mathbf{c}_{k}-\mu \mathbf{c}_{k}^{T} \boldsymbol{\gamma}_{k}+\frac{\mu}{\sigma_{0}^{2}} \mathbf{c}_{k}^{T} \mathbf{r}_{2}\left(\mathbf{q}_{k}\right) \\
-\frac{\mu^{2}}{2 \sigma_{n}^{2}} \mathbf{r}^{T} \mathbf{C}_{k} \mathbf{H} \mathbf{C}_{k} \mathbf{r}-\frac{\mu}{2 \sigma_{n}^{2}}\left(2 \mathbf{s}_{k}^{T} \mathbf{H}-\mathbf{b}\right) \mathbf{C}_{k} \mathbf{r} .
\end{gathered}
$$

Because of the definition of $z_{i}$ and $\mathbf{H}$ and with some manipulation the last term in the above equation $\left(\mu / 2 \sigma_{n}^{2}\right)\left(2 \mathbf{s}_{k}^{T} \mathbf{H}-\right.$ b) $\mathbf{C}_{k} \mathbf{r}$ is simplified as $\mu \mathbf{z}_{k}^{T} \mathbf{c}_{k}$. Replacement $\boldsymbol{\gamma}_{k}=-\mathbf{c}_{k}-\mathbf{z}_{k}+$ $\left(\mathbf{r}_{\mathbf{2}}\left(\mathbf{q}_{k}\right) / \sigma_{0}^{2}\right)$ from (75) into (77), results in (42).

\section{ACKNOWLEDGMENT}

The authors would like to thank the anonymous reviewers for their fruitful comments and suggestions.

\section{REFERENCES}

[1] D. L. Donoho, "For most large underdetermined systems of linear equations the minimal $l^{1}$-norm solution is also the sparsest solution," Comm. Pure Appl. Math, vol. 59, no. 6, pp. 797-829, 2006.

[2] R. Gribonval and M. Nielsen, "Sparse representations in unions of bases," IEEE Trans. Inf. Theory, vol. 49, no. 12, pp. 3320-3325, Dec. 2003.

[3] M. Zibulevsky and B. A. Pearlmutter, "Blind source separation by sparse decomposition in a signal dictionary," Neural Comput., vol. 13, no. 4, pp. 863-882, 2001.

[4] D. L. Donoho, M. Elad, and V. Temlyakov, "Stable recovery of sparse overcomplete representations in the presence of noise," IEEE Trans. Inf. Theory, vol. 52, no. 1, pp. 6-18, Jan. 2006.

[5] M. Davies and N. Mitianoudis, "Simple mixture model for sparse overcomplete ICA," in Proc. Inst. Electr. Eng._Vis. Image Signal Process., Feb. 2004, pp. 35-43.
[6] Y. Q. Li, S. Amari, A. Cichocki, D. W. C. Ho, and S. Xie, "Underdetermined blind source separation based on sparse representation," IEEE Trans. Signal Process., vol. 54, no. 2, pp. 423-437, Feb. 2006.

[7] P. G. Georgiev, F. J. Theis, and A. Cichocki, "Sparse component analysis and blind source separation of underdetermined mixtures," IEEE Trans. Neural Netw., vol. 16, pp. 992-996, Jul. 2005.

[8] R. Gribonval and S. Lesage, "A survey of sparse component analysis for blind source separation: Principles, perspectives, and new challenges," in Proc. Eur. Symp. Artificial Neuran Networks (ESANN), Apr. 2006, pp. 323-330.

[9] S. S. Chen, D. L. Donoho, and M. A. Saunders, "Atomic decomposition by basis pursuit," SIAM J. Sci. Comput., vol. 20, no. 1, pp. 33-61, 1999.

[10] J. L. Stark, M. Elad, and D. Donoho, "Image decomposition via the combination of sparse representations and a variational approach," IEEE Trans. Image Process., vol. 14, no. 10, pp. 1570-1582, Oct. 2005.

[11] M. Elad, R. Goldenberg, and R. Kimmel, "Low bit-rate compression of facial images," IEEE Trans. Image Process., vol. 16, no. 9, pp. 2379-2383, Sep. 2007.

[12] M. Elad and M. Aharon, "Image denoising via sparse and redundant representations over learned dictionaries," IEEE Trans. Image Process., vol. 15 , no. 12, pp. 3736-3745, 2006.

[13] O. G. Guleryuz, "Nonlinear approximation based image recovery using adaptive sparse reconstructions and iterated denoising-Part I: Theory," IEEE Trans. Image Process., vol. 15, no. 3, pp. 539-554, Mar. 2006.

[14] E. J. Candès and T. Tao, "Decoding by Linear Programming," IEEE Trans. Inf. Theory, vol. 51, no. 12, pp. 4203-4215, Dec. 2005.

[15] M. Akcakaya and V. Tarokh, "On sparsity, redundancy and quality of frame representations," in Proc. Int. Symp. Inf. Theory (ISIT), Jun. 2007, pp. 951-955

[16] H. Zayyani, M. Babaie-zadeh, and C. Jutten, "Decoding real-field codes by an iterative Expectation-Maximization (EM) algorithm," in Proc. IEEE Int. Conf. Acoustics, Speech, Signal Processing (ICASSP), Apr. 2008, pp. 3169-3172.

[17] D. L. Donoho, "Compressed sensing," IEEE Trans. Inf. Theory, vol 52, no. 4, pp. 1289-1306, Apr. 2006

[18] T. Blumensath and M. Davis, "Compressed sensing and source separation," in Proc. IEEE Int. Conf. Acoustics (ICA), Sep. 2007, pp 341-348.

[19] L. Vielva, D. Erdogmus, and C. Principe, "Underdetermined blind source separation using a probabilistic source sparsity model," in Proc. IEEE Int. Conf. Acoustics (ICA), 2001, pp. 675-679.

[20] C. Fevotte and S. J. Godsill, "A Bayesian approach for blind separation of sparse sources," IEEE Trans. Speech Audio Process., vol. 14, no. 6, pp. 2174-2188, Nov. 2006

[21] I. F. Gorodnitski and B. D. Rao, "Sparse signal reconstruction from limited data using FOCUSS: A re-weighted norm minimization algorithm," IEEE Trans. Signal Process., vol. 45, no. 3, pp. 600-616, Mar. 1997.

[22] M. Babaie-Zadeh, C. Jutten, and A. Mansour, "Sparse ICA via clusterwise PCA," Neurocomput., vol. 69, pp. 1458-1466, Aug. 2006.

[23] A. A. Amini, M. Babaie-Zadeh, and C. Jutten, "A fast method for sparse component analysis based on iterative detection-projection," in MaxEnt'06, AIP Conf. Proc., 2006, vol. 872, pp. 123-130.

[24] H. Zayyani, M. Babaie-Zadeh, and C. Jutten, "Source estimation in noisy sparse component analysis," in Proc. 15th Int. Conf. Digital Signal Processing (DSP), Jul. 2007, pp. 219-222.

[25] G. H. Mohimani, M. Babaie-Zadeh, and C. Jutten, "A fast approach for overcomplete sparse decomposition based on smoothed $\ell^{0}$ norm," IEEE Trans. Signal Process., vol. 57, no. 1, pp. 289-301, Jan. 2009.

[26] G. H. Mohimani, M. Babaie-zadeh, and C. Jutten, " Fast sparse representation based on smoothed $\ell^{0}$-norm," in Proc. IEEE Int. Conf. Acoustics (ICA), Sep. 2007, pp. 389-396.

[27] H. Zayyani, M. Babaie-Zadeh, G. H. Mohimani, and C. Jutten, "Sparse component analysis in presence of noise using an iterative EM-MAP algorithm," in Proc. IEEE Int. Conf. Acoustics (ICA), Sep. 2007, pp. $438-445$.

[28] S. Mallat and Z. Zhang, "Matching pursuits with time-frequency dictionaries," IEEE Trans. Signal Process., vol. 41, no. 12, pp. 3397-3415, Dec. 1993.

[29] M. Lewicki and T. J. Sejnowski, "Learning overcomplete representations," Neural Comput., vol. 12, pp. 337-365, 2000. 
[30] S. Krstulovic and R. Gribonval, "MPTK: Matching pursuit made tractable," in Proc. IEEE Int. Conf. Acoustics, Speech, Signal Processing (ICASSP), May 14-19, 2006, pp. 496-499.

[31] Y. C. Pati, R. Rezaiifar, and P. S. Krishnaprasad, "Orthogonal matching pursuit: Recursive function approximation with application to wavelet decomposition," in Conf. Rec. 27th Asilomar Conf. Signals, Syst. Comput., 1993, pp. 40-44.

[32] D. L. Donoho, Y. Tsaig, I. Drori, and J. L. Starck, "Sparse solution of underdetermined linear equations by stagewise orthogonal matching pursuit,” Tech. Rep., 2006 [Online]. Available: http://www-stat.stanford.edu/ idrori/STOMP.pdf

[33] D. Wipf and B. D. Rao, "Sparse Bayesian learning for basis selection," IEEE Trans. Signal Process., vol. 52, no. 8, pp. 2153-2164, Aug. 2004.

[34] S. Ji, Y. Xue, and L. Carin, "Bayesian compressive sensing," IEEE Trans. Signal Process., vol. 56, no. 6, pp. 2346-2356, Jun. 2008.

[35] T. Blumensath and M. Davies, "Gradient pursuits," IEEE Trans. Signal Process., vol. 56, no. 6, pp. 2370-2382, Jun. 2008.

[36] M. A. T. Figueirado, R. D. Nowak, and S. J. Wright, "Gradient projection for sparse reconstruction: Application to compressed sensing and other inverse problems," IEEE J. Sel. Topics Signal Process. 2007 [Online]. Available: http://www.ece.wisc.edu/ nowak/GPSR.pdf, to be published

[37] A. Dempster, N. Laird, and D. Rubin, "Maximum likelihood estimation from incomplete data via the EM algorithm," J. Roy. Statist. Soc. B, vol. 39, pp. 1-38, 1977.

[38] M. A. T. Figueirado and R. D. Nowak, "An EM algorithm for waveletbased image restoration," IEEE Trans. Image Process., vol. 12, no. 8, pp. 906-916, Aug. 2003.

[39] J. M. Mendel, Maximum Likelihood Deconvolution. New York: Springer, 1990.

[40] H. L. Taylor, S. C. Banks, and J. F. McCoy, "Deconvolution with the $\ell^{1}$-norm," Geophys., vol. 44, no. 1, pp. 39-52, 1979.

[41] J. J. Kormylo and J. M. Mendel, "Maximum likelihood detection and estimation method for sparse deconvolution," IEEE Trans. Inf. Theory, vol. 28, no. 3, pp. 482-488, May 1982.

[42] M. Lavielle, "Bayesian deconvolution of Bernoulli-Gaussian processes," Signal Process., vol. 33, no. 1, pp. 67-79, Jul. 1993.

[43] I. Santamaria-Caballero, C. J. Pantaleon-Prieto, and A. Artes-Rodriguez, "Sparse deconvolution using adaptive mixed-Gaussian models," Signal Process., vol. 54, pp. 161-172, 1996.

[44] A. M. Djafari, "Bayesian source separation: Beyond PCA and ICA," in Proc. Eur. Symp. Artificial Neuran Networks (ESANN), Apr. 2006, pp. 313-322.

[45] F. Labeau, J. C. Chiang, M. Kieffer, P. Duhamel, L. Vandendorpe, and B. Macq, "Oversampled filter banks as error correcting codes: Theory and impulse noise cancellation," IEEE Trans. Signal Process., vol. 53, no. 12, pp. 4619-4630, Dec. 2005.

[46] E. Larsson and Y. Selen, "Linear regression with a sparse parameter vector," IEEE Trans. Signal Process., vol. 55, no. 2, pp. 451-460, Feb. 2007.

[47] A. M. Tomasi, Estimating Gaussian Mixture Density With EM-A Tutorial [Online]. Available: http://www.cs.duke.edu/courses/spring04/ cps196.1/handouts/EM/tomasiEM.pdf

[48] B. D. Anderson and J. B. Moore, Optimal Filtering. Englewood Cliffs, NJ: Prentice-Hall, 1979.

[49] A. Blake and A. Zisserman, Visual Reconstruction. Cambridge, MA: MIT Press, 1987.

[50] E. J. Candès and J. Romberg, $\ell^{1}$-Magic: Recovery of Sparse Signals Via Convex Programming, 2005 [Online]. Available: http://www.acm. caltech.edu/l1magic/downloads/11-magic.pdf

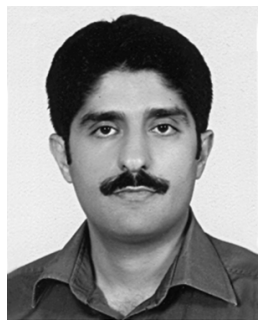

Hadi Zayyani (S'09) was born in Shiraz, Iran, in 1978. He received the B.S. and M.S. degrees, both in electrical engineering (communications), in 2001 and 2003, respectively, from Sharif University of Technology, Tehran, Iran, where he is currently working towards the Ph.D. degree.

His research interests include statistical signal processing, sparse component analysis, sparse signal representation and its applications, dictionary learning, and real-field coding.

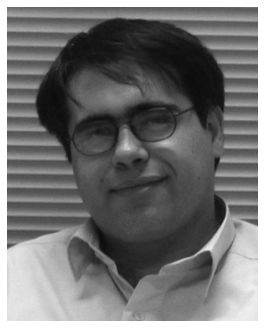

Massoud Babaie-Zadeh (M'04-SM'09) received the B.S. degree in electrical engineering from Isfahan University of Technology, Isfahan, Iran, in 1994, the M.S. degree in electrical engineering from Sharif University of Technology, Tehran, Iran, in 1996, and the Ph.D. degree in signal processing from the Institute National Polytechnique of Grenoble (INPG), Grenoble, France, in 2002.

Since 2003, he has been a faculty member of the Electrical Engineering Department of Sharif University of Technology, Tehran, Iran, first as an Assistant Professor, and since 2008 as an Associate Professor. His main research interests are blind source separation (BSS) and independent component analysis (ICA), sparse signal processing, and statistical signal processing.

Dr. Babaie-Zadeh received the best Ph.D. thesis award of INPG for his Ph.D. dissertation.

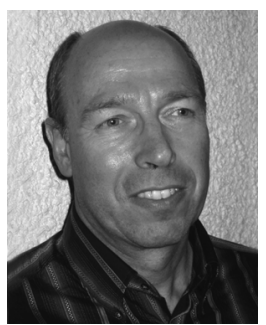

Christian Jutten (AM'92-M'03-SM'06-F'08) received the Ph.D. degree and the Docteurès Sciences degree both from the Institut National Polytechnique of Grenoble, Grenoble, France, in 1981 and 1987, respectively.

He taught as Associate Professor in the Ecole Nationale Supérieure d'Electronique et de Radioélectricité of Grenoble, France, from 1982 to 1989 . He was Visiting Professor in the Swiss Federal Polytechnic Institute, Lausanne, Switzerland, in 1989, before becoming Full Professor in the Université Joseph Fourier of Grenoble, France, more precisely in the Polytech'Grenoble institute. He is currently Associate Director of the images and signals laboratory. For 25 years, his research interests have focused on blind source separation, independent component analysis and learning in neural networks, including theoretical aspects (separability, source separation in nonlinear mixtures), applications in signal processing (biomedical, seismic, speech), and data analysis. $\mathrm{He}$ is author or coauthor of more than 40 papers in international journals, 16 invited papers, and 100 communications in international conferences.

Dr. Jutten has been Associate Editor of the IEEE TRANSACTIONS ON CiRCUITS AND SYSTEMS from 1994 to 1995, and co-organizer with Dr. J.-F. Cardoso and Prof. Ph. Loubaton of the First International Conference on Blind Signal Separation and Independent Component Analysis, Aussois, France, January 1999. He is currently a member of a technical committee of the IEEE Circuits and Systems society on blind signal processing. He is a reviewer of main international journals (IEEE TRANSACTIONS ON SignAL PROCESSING, IEEE Signal Processing LetTers, IEEE Transactions on Neural Networks, Signal Processing, Neural Computation, Neurocomputing, etc.) and conferences in signal processing and neural networks (ICASSP, ISCASS, EUSIPCO, IJCNN, ICA, ESANN, IWANN, etc.). 